EUROPEAN ORGANIZATION FOR NUCLEAR RESEARCH

European Laboratory for Particle Physics

\title{
A Study of the Coherent Beam-Beam Effect in the Framework of the Vlasov Perturbation Theory
}

\author{
Y. Alexahin*
}

\begin{abstract}
A number of factors which can influence coherent beam-beam oscillations are studied on the basis of the Vlasov equation: difference in the intensities and single-particle tunes in the beams; difference in the phase advances between interaction points; long-range interactions; synchro-betatron coupling due to betatron phase advance variation in the vicinity of IP, chromatic tune modulation and crossing angle.

The synchro-betatron coupling appears to have a principal stabilizing effect: at synchrotron tune values in the neighborhood of half the beam-beam parameter it provides Landau damping of the discrete spectral lines by overlapping sidebands; the damping rate being higher with negative chromaticity. At smaller synchrotron tune values a specific mode of the head-tail damping sets in which is not sensitive to chromaticity.

Application to LHC shows that with the design values of parameters the coherent beam-beam oscillations should be Landau damped, probably with the help of negative chromaticity of a moderate absolute value.
\end{abstract}

Submitted to "Nuclear Instruments \& Methods in Physics Research. Series A"

*Permanent address: PPL JINR, Dubna 141980 Russia; e-mail: alexahin@sunse.jinr.ru, alexahin@mail.cern.ch

Administrative Secretariat

LHC Division

CERN

CH-1211 Geneva 23

Switzerland

Geneva , 29 January 2001 


\section{Contents}

1 Introduction 3

2 Green's function of the beam-beam potential 4

3 Equilibrium state 5

3.1 Nonlinear normal forms 5

$\begin{array}{lll}3.2 & \text { Head-tail phase } & 7\end{array}$

$\begin{array}{ll}3.3 & \text { Normalized action-angle variables }\end{array}$

4 The Vlasov equation 7

4.1 Fourier expansion in the angle variables 9

$5 \quad$ Spectrum of uncoupled modes 10

5.1 Spectral density of dipole oscillations 11

5.2 Effect of tunesplit and unequal intensities $\quad 12$

5.3 Effect of redistribution of phase advances 13

$6 \quad$ Long-range interactions $\quad 15$

$\begin{array}{lll}6.1 & \text { Multi-bunch modes } & 15\end{array}$

6.2 Effect of lumped long-range interactions 17

7 Coherent beam-beam resonances 18

8 Influence of synchrotron motion on coherent beam-beam modes 20

8.1 Effect of finite bunch length and chromaticity 20

8.1.1 High synchrotron tune 21

8.1.2 Low synchrotron tune 22

8.1.3 Intermediate synchrotron tune 23

8.2 Effect of crossing angle $\quad 24$

8.3 Implications for LHC 25

9 Summary 26

$\begin{array}{ll}\text { Acknowledgements } & 27\end{array}$

$\begin{array}{ll}\text { References } & 27\end{array}$

$\begin{array}{lll}\text { Appendix A. Landau damping by sidebands } & 28\end{array}$

A.1 Landau recipe $\quad 28$

A.2 Van Kampen approach $\quad 30$

A.3 Correspondence of the two methods 31

Appendix B. Beam-beam effect on the slow head-tail instability 32 


\section{Introduction}

In high-luminosity hadron colliders operating in the $\mathrm{TeV}$ energy range the coherent beam dynamics is dominated by the beam-beam interaction. Its nonlinearity introduces large spreads in the incoherent tunes, exceeding by many times the threshold value needed for suppression of instabilities that could originate from the beam-wall interaction.

At the same time the opposing beam acts as a reactive impedance shifting tunes of coherent oscillations. In the case of equal tunes and intensities the coherent tune shift is larger than the maximum incoherent tune shift giving rise to the discrete spectral lines of coherent oscillations [1].

When the discrete lines are well separated from the continuum of incoherent tunes the beam-beam interaction not only fails to produce damping of coherent oscillations but can even switch off other stabilizing mechanisms. Therefore it is important to better understand what factors influence the coherent beam-beam modes, why they are seen in some situations but clearly absent in the others.

First we analyze spectra of uncoupled modes of coherent oscillations. There is a number of mechanisms which reduce coherence of the beam-beam oscillations bringing the discrete spectral lines closer to (or even within) the incoherent tune spread.

A new look is taken at already known effects of unbalance in the beam intensities [2] and split in the single particle tunes $[3,4]$ and their interference. The explanation is given to the suppression of coherent beam-beam oscillations, observed in simulation [5], by $\pi / 2$-phase advance redistribution between two diametrically opposite interaction points (IPs).

Multiple long-range interactions are considered in the context of the rigid-bunch model prediction of the possibility to reverse the coherent tune shift in the plane of separation by cumulative action of a sufficient number of such interactions.

However, in the case of a large tune split between the beams the mode coupling can lead to excitation of coherent beam-beam resonances of relatively low order, turning the beam-beam interaction itself into a source of instability. Such possibility is also briefly discussed in this paper.

The major attention is devoted to the influence of synchro-betatron coupling on the coherent oscillations. Different mechanisms can bring about such coupling: betatron phase advance variation along the bunches at IP, which we will refer to as just " the finite bunch length effect" for the sake of brevity; chromatic tune modulation; crossing angle.

All these mechanisms produce some dephasing of betatron oscillations leading to a reduction in coherent beam-beam tune shift. But what is even more important, the synchrotron sidebands of incoherent tunes can overlap the discrete spectral lines providing Landau damping.

In order to analyze the effect of these factors in the framework of the self-consistent kinetic theory some methodical development is necessary. In Section 2 we derive the Hamiltonian of a particle interaction with a bunch of finite length and arbitrary charge distribution. In Section 3 we demonstrate with the help of the Lie transform method how the so-called chromatic head-tail phase appears in the result of the single particle Hamiltonian normalization. Derivation of the Landau damping rate due to sideband overlap (with discussion of different approaches) is relegated to Appendix A.

It should be noted that analysis in the present paper is limited to the first order effects, not only in the deviation of the distribution function from the equilibrium, but in the beambeam parameter as well. Also, throughout the paper the emittances of the beams (and optical functions at IPs in the case of two rings) are assumed equal. 

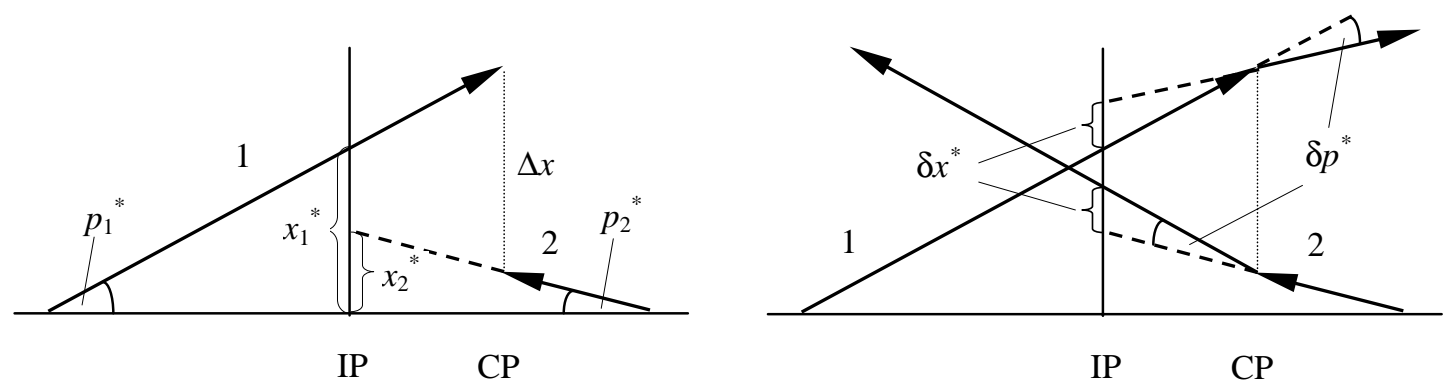

Figure 1. Sketch of the interaction of two particles.

\section{Green's function of the beam-beam potential}

Let us make the conventional choice of the generalized azimuth $\theta=s / R$ as the independent variable (so that the longitudinal momentum plays the role of the Hamiltonian) and introduce canonically conjugated coordinates and momenta $\underline{q}=(x, y, \sigma), \underline{p}=\left(p_{x}, p_{y}, \delta_{p}\right)$, where in the ultra-relativistic case $\sigma=s-c t$.

In fact we will understand $\theta$ as normalized time which is common to both beams, whereas the arc length $s$ is individual for each beam (certainly the mean radii $R$ of both orbits are assumed to be equal).

Now let us consider the interaction of two particles belonging to the counter-rotating bunches. Due to the synchrotron motion the actual collision point (CP) is shifted w.r.t. the interaction point (IP) of the reference particles by the amount

$$
s_{1}=-s_{2}=\frac{\sigma_{1}-\sigma_{2}}{2} .
$$

However, limiting ourselves to first order effects in the beam-beam force, we can noticeably simplify the problem by ascribing the interaction to the position of the reference IP (the moment of "time" $\theta=\theta_{I P}$ ) by a similarity transformation [6] which is explained in Fig.1. Up to the second order in the momenta the actual distance between the particles at CP is expressed via their coordinates and momenta at IP (marked with an asterisk) as

$$
\Delta x=\left(x_{1}^{*}+p_{1}^{*} s_{1}\right)-\left(x_{2}^{*}+p_{2}^{*} s_{2}\right)=x_{1}^{*}-x_{2}^{*}+\frac{p_{1}^{*}+p_{2}^{*}}{2}\left(\sigma_{1}-\sigma_{2}\right) .
$$

The variation in momenta due to the interaction is

$$
\delta p_{1}^{*}=-\delta p_{2}^{*}=\frac{2 r_{p}}{\gamma \Delta x}
$$

where $r_{p}$ is the classical radius of the particles ( $r_{p}>0$ if the charges of the particles have the same sign and $r_{p}<0$ otherwise), $\gamma$ is the relativistic mass factor.

By ascribing the interaction to the reference IP, we should admit a jump in the particle coordinates (see Fig.1 right) by

$$
\delta x_{1}^{*}=\delta x_{2}^{*}=-\delta p_{1}^{*} \frac{\sigma_{1}-\sigma_{2}}{2} .
$$

The variations (2.3), (2.4) can be obtained from the elementary interaction Hamiltonian 


$$
H=-\frac{2 r_{p}}{\gamma} \delta\left(\theta-\theta_{I P}\right) \ln \left[x_{1}-x_{2}+\frac{p_{1}+p_{2}}{2}\left(\sigma_{1}-\sigma_{2}\right)\right]
$$

(which gives a correct value of the energy exchange as well). Correspondingly, the interaction of a particle of the $k$-th beam $(k=1,2)$ with the whole of the counter-rotating bunch can be described by the Hamiltonian,

$$
H_{b b}^{(k)}=\frac{r_{p} N_{3-k}}{\gamma} \delta_{\mathrm{p}}\left(\theta-\theta_{I P}\right) \int G\left(\underline{q}, \underline{p} \mid \underline{q^{\prime}}, \underline{p^{\prime}}\right) F^{(3-k)}\left(\underline{q^{\prime}}, \underline{p^{\prime}}, \theta\right) d^{3} q^{\prime} d^{3} p^{\prime},
$$

$\delta_{\mathrm{p}}(\theta)$ is the periodic $\delta$-function, $N_{k}$ is the number of particles per bunch and $F^{(k)}$ is the distribution function of the $k$-th beam normalized to unity.

Taking into account also the vertical plane we can write for the Green function under the assumptions made

$$
G=-\ln \left\{\left[x-x^{\prime}+\frac{p_{x}+p_{x}^{\prime}}{2}\left(\sigma-\sigma^{\prime}\right)\right]^{2}+\left[y-y^{\prime}+\frac{p_{y}+p_{y}^{\prime}}{2}\left(\sigma-\sigma^{\prime}\right)\right]^{2}\right\} .
$$

By $p_{x, y}$ we understand here the full slopes of the trajectories which may include crossing angles (see next Section). Taking them out explicitly would cast the Green function (2.7) into the form presented (without derivation) in Ref.[7].

\section{Equilibrium state}

Let us postulate that there exists the object of stability analysis, an equilibrium state. This requires that in the absence of perturbation the motion of (almost) all the particles is stable.

We will make the even stronger assumption (not necessary in principle) that the particle motion is regular in the relevant volume of the phase space. This is indeed a quite strong requirement in the presence of nonlinear beam-beam force. It means that invariants of motion $\underline{I}=\left(I_{x}, I_{y}, I_{s}\right)$ exist which can be used in the construction of the equilibrium distribution function which we presume to be Gaussian:

$$
F_{0}=\frac{1}{(2 \pi)^{3} V} \exp \left(-\underline{\varepsilon^{-1}} \cdot \underline{I}\right), \quad \underline{\varepsilon}=\langle\underline{I}\rangle, \quad V=\varepsilon_{x} \varepsilon_{y} \varepsilon_{s}, \quad \underline{\varepsilon^{-1}}=\left(\varepsilon_{x}^{-1}, \varepsilon_{y}^{-1}, \varepsilon_{s}^{-1}\right) .
$$

\section{General remark:}

The invariants $\underline{I}$ should be found in a self-consistent way with account of the equilibrium beam-beam potential. Here we encounter a minor complication since the distribution function in the original variables is not known yet. However, the distribution function appears only in the integrand so the potential should not be very sensitive to its form permitting an iterative procedure. Anyway, the error is in the second order in the beam-beam parameter which we ignore in the present study.

\subsection{Nonlinear normal forms}

Before starting search for the nonlinear invariants, $\underline{I}$, let us introduce linear normal form variables, $\underline{a}$, related to coordinates and momenta by formulas ${ }^{1}$ (see e.g. Ref.[8], p.294)

$$
\begin{array}{ll}
x=\sqrt{\frac{\beta_{x}}{2}} e^{i \phi_{x}} a_{x}+\text { c.c., } & p_{x}=\frac{i-\alpha_{x}}{\sqrt{2 \beta_{x}}} e^{i \phi_{x}} a_{x}+\text { c.c., }, . . \\
\sigma=\sqrt{\frac{\beta_{s}}{2}} a_{s}+\text { c.c., } & \delta_{p}=\frac{i}{\sqrt{2 \beta_{s}}} a_{s}+\text { c.c. },
\end{array}
$$

\footnotetext{
${ }^{1}$ Here and afterwards we write down formulas for the horizontal plane only.
} 
where $\alpha_{x}, \beta_{x}$ are the Twiss parameters of the lattice, $\phi_{x}=\mu_{x}(\theta)-v_{x 0} \cdot \theta, \mu_{x}$ and $v_{x 0}$ being the betatron phase advance and the single particle tune, $\beta_{s}=\left|\alpha_{M} R / v_{s}\right|, \alpha_{M}$ and $v_{s}$ being the momentum compaction factor and the synchrotron tune ( $v_{s}<0$ above transition).

The linear normal form variables satisfy the Hamilton equations

$$
\frac{d}{d \theta} a_{j}=\frac{\partial}{\partial \bar{a}_{j}} U, \quad \frac{d}{d \theta} \bar{a}_{j}=-\frac{\partial}{\partial a_{j}} U, \quad j=x, y, s,
$$

where the bar means complex conjugation, with imaginary Hamiltonian

$$
U=U_{0}+U_{1}, \quad U_{0}=i \sum_{j} v_{j 0} a_{j} \bar{a}_{j}, \quad U_{1}=i H_{1},
$$

where $H_{1}$ comprises contribution from the lattice nonlinearities and the beam-beam potential.

Our aim is to find new, nonlinear normal form variables, $A_{j}=\hat{T} a_{j}$, such that the transformed Hamiltonian $V$ would have the form

$$
V=i \sum_{j} \mathrm{v}_{j} A_{j} \bar{A}_{j}
$$

with new tunes $v_{j}$, possibly depending on the integrals of motion,

$$
I_{j}=\left|A_{j}\right|^{2},
$$

we are looking for. Introducing the phase angles via relations

$$
A_{j}=\sqrt{I_{j}} e^{i \psi_{j}}
$$

we would obtain the solution of the Hamilton equations in the form $I_{j}=$ const, $\psi_{j}=v_{j} \theta$.

There is an efficient method of the Hamiltonian normalization, called Deprit's algorithm, via a Lie-transform. We refer the reader to the excellent book [8] for details and give here only a few formulas necessary for the following.

The new Hamiltonian to the first order in $U_{1}$ is

$$
V=V_{0}+V_{1}, \quad V_{0}=i \sum_{j} v_{j 0} A_{j} \bar{A}_{j}, \quad V_{1}=<U_{1}>
$$

where the brackets mean implementation of the following recipe: substitute (3.7) for $a_{j}$ and then perform averaging over $\psi_{j}$ and $\theta$.

The first order generating function of the Lie-transform $w_{1}$ satisfies the homology equation

$$
\frac{\partial}{\partial \theta} w_{1}+\left[w_{1}, V_{0}\right]=V_{1}-U_{1}
$$

where the Poisson brackets were introduced,

$$
[f, g] \equiv \sum_{j}\left(\frac{\partial f}{\partial A_{j}} \frac{\partial g}{\partial \bar{A}_{j}}-\frac{\partial f}{\partial \bar{A}_{j}} \frac{\partial g}{\partial A_{j}}\right)
$$

and $U_{1}$ must be again formally understood as a function of the new variables, $\underline{A}$.

As long as $w_{1}$ commutes with $U_{1}$ (e.g. when $U_{1}$ contains just one term) no higher order terms arise and the operator of the inverse Lie-transform can be given in the closed form

$$
\hat{T}^{-1}=e^{-\hat{L}\left(w_{1}\right)}, \quad \hat{L}(f) g \equiv-[f, g] .
$$




\subsection{Head-tail phase}

Let us show how the so-called head-tail phase (see e.g. Ref.[9]) appears from the term in the Hamiltonian which describes the chromatic dependence of the betatron tune and in the original variables has the form

$$
H_{1}=v_{x}^{\prime} \delta_{p} I_{x}^{(0)}=\frac{i v_{x}^{\prime}}{\sqrt{2 \beta_{s}}}\left(a_{s}-\bar{a}_{s}\right) a_{x} \bar{a}_{x},
$$

where $v_{x}^{\prime}=d v_{x} / d \delta_{p}$

In this case $V_{1}=0$ and eq.(3.9) gives

$$
w_{1}=i \frac{v_{x}^{\prime}}{\sqrt{2 \beta_{s} \mid}\left|v_{s}\right|}\left(A_{s}+\bar{A}_{s}\right) A_{x} \bar{A}_{x} .
$$

The inverse Lie-transform (3.11) reproduces the well-known result for the head-tail phase

$$
a_{x}=\hat{T}^{-1} A_{x}=\exp \left[-\frac{i{\nu_{x}^{\prime}}^{\prime}}{\sqrt{2 \beta_{s}}\left|\nu_{s}\right|}\left(A_{s}+\bar{A}_{s}\right)\right] A_{x} \approx A_{x} \exp \left[-\frac{i{V_{x}^{\prime}}^{\prime}}{\alpha_{M} R} \sigma\right] .
$$

\subsection{Normalized action-angle variables}

In the following we will use the new action-angle variables $\underline{I}=\left(I_{x}, I_{y}, I_{s}\right), \underline{\Psi}=\left(\Psi_{x}, \psi_{y}, \Psi_{s}\right)$ related to the normal forms by eqs.(3.6), (3.7). In these variables the normalized Hamiltonian can be written as

$$
K_{0}^{(k)}(\underline{I})=\underline{v}_{0}^{(k)} \cdot \underline{I}+\left\langle H_{b b}^{(k)}\right\rangle, \quad k=1,2,
$$

where we made provision for different parameters of the two beams, the subscript " 0 " at the Hamiltonian now means the steady state value (absence of coherent perturbations).

In the steady state the action-angle variables satisfy the following equations of motion

$$
\underline{I}=0, \quad \underline{\dot{\psi}}=\underline{\mathbf{v}}^{(k)}(\underline{I}) \equiv \underline{\mathbf{v}}_{0}^{(k)}+\underline{\mathbf{v}}_{b b}^{(k)}, \quad \underline{\mathbf{v}}_{b b}^{(k)}=\frac{\partial}{\partial \underline{I}}\left\langle H_{b b}^{(k)}\right\rangle .
$$

Allowing for the horizontal crossing angle, $2 \alpha$, and beam-beam offsets $d_{x}$ (and $d_{y}$ in the formulas for the vertical plane) we can express the physical coordinates and momenta of particles in $k$-th beam at a given interaction point via the new variables as follows

$$
\begin{aligned}
& x=(-1)^{k-1} d_{x} / 2+\sqrt{2 \beta_{x}^{*} I_{x}} \sin \left[\psi_{x}+\phi_{x}^{(k)}\left(\theta_{I P}\right)-\chi \sigma\right], \\
& p_{x}=\alpha+\sqrt{2 I_{x} / \beta_{x}^{*}} \cos \left[\psi_{x}+\phi_{x}^{(k)}\left(\theta_{I P}\right)-\chi \sigma\right], \\
& \sigma=\sqrt{2 \beta_{s} I_{s}} \sin \psi_{s}, \quad \delta_{p}=\sqrt{2 I_{s} / \beta_{s}} \cos \psi_{s},
\end{aligned}
$$

where we have assumed the betatron function to have no slope at the IP $\left(\alpha_{x}^{*}=0\right)$, and introduced the chromaticity related parameter

$$
\chi=v_{x}^{\prime} /\left(\alpha_{M} R\right) \text {. }
$$

\section{The Vlasov equation}

Let us consider now a small perturbation of the steady state and present the distribution function of the $k$-th beam as the sum

$$
F^{(k)}=F_{0}+F_{1}^{(k)} \text {. }
$$

Correspondingly, we have for the Hamiltonian 


$$
\begin{aligned}
& K^{(k)}=K_{0}^{(k)}+K_{1}^{(k)}, \\
& K_{1}^{(k)}(\underline{I}, \underline{\psi})=\sum_{I P} \frac{r_{p} N_{3-k}}{\gamma} \delta_{\mathrm{p}}\left(\theta-\theta_{I P}\right) \int G^{(k)} F_{1}^{(3-k)}\left(\underline{I^{\prime}}, \underline{\Psi^{\prime}}\right) d^{3} I^{\prime} d^{3} \psi^{\prime} .
\end{aligned}
$$

where the Green function is given by eq.(2.7) in which coordinates and momenta should be expressed via the action-angle variables with the help of eqs.(3.17). The superscript $k$ has appeared since there is an explicit dependence of the Green function on the beam number in the case of finite offsets and/or phase advance deviations.

Linearizing the Liouville equations for the two beams $(k=1,2)$

$$
\frac{\partial}{\partial \theta} F^{(k)}+\left[F^{(k)}, K^{(k)}\right]=0
$$

w.r.t. $F_{1}$ we come to (the system of) the Vlasov equations

$$
\frac{\partial}{\partial \theta} F_{1}^{(k)}+\underline{v}^{(k)} \cdot \frac{\partial}{\partial \underline{\psi}} F_{1}^{(k)}=-F_{0} \underline{\varepsilon^{-1}} \cdot \frac{\partial}{\partial \underline{\psi}} K_{1}^{(k)}(\underline{I}, \underline{\psi} ; \theta) .
$$

Allowing for unequal intensities (without loss of generality we may assume the first beam to be the weaker one, $\left.r \xi=N_{1} / N_{2} \leq 1\right)$ let us introduce the vector of normalized distribution functions

$$
\mathbf{f}=e^{\varepsilon^{-1} \cdot \underline{I}} / 2\left(\begin{array}{c}
\sqrt{r_{\xi}} F_{1}^{(1)} \\
F_{1}^{(2)}
\end{array}\right)
$$

and rewrite eq.(4) in the matrix form

$$
i \frac{\partial}{\partial \theta} \mathbf{f}=\hat{A}(\theta) \cdot \mathbf{f}
$$

where the following operators were introduced

$$
\begin{aligned}
& \hat{A}=\left(\begin{array}{cc}
\underline{v}^{(1)} \cdot \underline{\hat{D}} & 0 \\
0 & \underline{v}^{(2)} \cdot \underline{\hat{D}}
\end{array}\right)+\underline{\varepsilon^{-1}} \cdot \underline{\hat{D}} \sum_{I P} \frac{r_{p}}{\gamma} \sqrt{N_{1} N_{2}} \delta_{\mathrm{p}}\left(\theta-\theta_{I P}\right)\left(\begin{array}{cc}
0 & \hat{G}^{(1)} \\
\hat{G}^{(2)} & 0
\end{array}\right), \\
& \underline{\hat{D}}=-i \frac{\partial}{\partial \underline{\psi}}, \\
& \hat{G}^{(k)} f=\frac{1}{(2 \pi)^{3} V} \int e^{-\underline{\varepsilon^{-1}} \cdot\left(\underline{I}+\underline{I}^{\prime}\right) / 2} G^{(k)}\left(\underline{I}, \underline{\Psi} \mid \underline{I^{\prime}}, \underline{\psi^{\prime}}\right) f\left(\underline{I^{\prime}}, \underline{\psi^{\prime}}\right) d^{3} I^{\prime} d^{3} \psi^{\prime} .
\end{aligned}
$$

In the following we will refer to $\hat{A}$ as the Vlasov operator and call its second term the coherent part (in contradistinction to the first term which incorporates the incoherent beambeam tune shifts).

Let us have a look into the properties of the Green function in more detail. Recalling (3.17) and allowing for a finite crossing angle we can present the horizontal distance (2.2) between the particles as ${ }^{2}$

$$
\begin{aligned}
\Delta x= & (-1)^{k-1} d_{x}+\sigma_{x} \sqrt{2\left(1+b^{2}\right)}\left\{\sqrt{J_{x}} \sin \left[\psi_{x}+\phi_{x}^{(k)}\left(\theta_{I P}\right)-\chi \sigma-\varphi\right]-\right. \\
& \left.-\sqrt{J_{x}^{\prime}} \sin \left[\psi_{x}^{\prime}+\phi_{x}^{(3-k)}\left(\theta_{I P}\right)-\chi \sigma^{\prime}+\varphi\right]\right\}+\alpha\left(\sigma-\sigma^{\prime}\right),
\end{aligned}
$$

where the following notations were introduced

\footnotetext{
${ }^{2}$ Please note the different signs at $\varphi$, which we will refer to as "the beam-beam head-tail phase".
} 


$$
\begin{aligned}
& \sigma_{i}=\sqrt{\beta_{i} \varepsilon_{i}}, \quad J_{i}=I_{i} / \varepsilon_{i}, \quad i=x, y, s, \\
& b=\frac{\sigma-\sigma^{\prime}}{2 \beta_{x}^{*}}=\frac{\sigma_{s}}{\sqrt{2} \beta_{x}^{*}}\left(\sqrt{J_{s}} \sin \psi_{s}-\sqrt{J_{s}^{\prime}} \sin \psi_{s}^{\prime}\right), \\
& \varphi=-\arctan b .
\end{aligned}
$$

In the round beam case, the phase $\varphi$ is the same for both transverse planes which makes it similar to the case of horizontal oscillations in flat beams in respect to the finite bunch length effect.

\section{General remark:}

It can be easily verified that the Green function has the following symmetry

$$
G^{(k)}\left(\underline{J}, \underline{\Psi} \mid \underline{J^{\prime}}, \underline{\psi^{\prime}}\right)=G^{(3-k)}\left(\underline{J^{\prime}}, \underline{\psi^{\prime}} \mid \underline{J}, \underline{\Psi}\right)
$$

so that the matrix operator in the coherent part of $\hat{A}$ is self-adjoint. The operator $\underline{\hat{D}}$ is also self-adjoint on periodic functions of $\Psi$. However their composition is not necessarily self-adjoint since in general these operators do not commute. Therefore the spectrum of the Vlasov operator $\hat{A}$ is not necessarily real which implies possible instability due to the beam-beam interaction.

\subsection{Fourier expansion in the angle variables}

To reduce the dimensionality of the problem let us perform a Fourier expansion in the angle variables

$$
\mathbf{f}(\underline{I}, \underline{\psi}, \theta)=\sum_{\underline{m}} \exp (i \underline{m} \cdot \underline{\psi}) \mathbf{f}_{\underline{m}}(\underline{I}, \theta)
$$

where $\underline{m}=\left(m_{x}, m_{y}, m_{s}\right)$ are 3-tuples of integer numbers. Then from eq.(6) we obtain the following system of integral equations for the Fourier amplitudes

$$
i \frac{\partial}{\partial \theta} \mathbf{f}_{\underline{m}}=\left(\begin{array}{cc}
\underline{m} \cdot \underline{\underline{v}}^{(1)} & 0 \\
0 & \underline{m} \cdot \underline{v^{(2)}}
\end{array}\right) \cdot \mathbf{f}_{\underline{m}}+\underline{m} \cdot \underline{\varepsilon^{-1}} \sum_{I P} \frac{r_{p}}{\gamma} \sqrt{N_{1} N_{2}} \delta_{\mathrm{p}}\left(\theta-\theta_{I P}\right) \sum_{\underline{\underline{m}^{\prime}}}\left(\begin{array}{cc}
0 & \hat{G}_{\underline{m} \underline{m}^{\prime}}^{(1)} \\
\hat{G}_{\underline{m} \underline{m}^{\prime}}^{(2)} & 0
\end{array}\right) \cdot \mathbf{f}_{\underline{m^{\prime}}}
$$

where the integral operators are now defined as follows

$$
\begin{aligned}
& \hat{G}_{\underline{\underline{m} \underline{m}^{\prime}}}^{(k)} f=\int \exp \left[-\sum_{i}\left(J_{i}+J_{i}^{\prime}\right) / 2\right] G_{\underline{m} \underline{m^{\prime}}}^{(k)}\left(\underline{J}, \underline{J^{\prime}}\right) f\left(\underline{J^{\prime}}\right) d^{3} J^{\prime}, \\
& G_{\underline{m} \underline{m}^{\prime}}^{(k)}\left(\underline{J}, \underline{J^{\prime}}\right)=\frac{1}{(2 \pi)^{6}} \int_{0}^{2 \pi} G^{(k)}\left(\underline{J}, \underline{\Psi} \mid \underline{J^{\prime}}, \underline{\psi^{\prime}}\right) e^{-i \underline{m} \cdot \underline{\psi}+i \underline{m^{\prime}} \cdot \underline{\psi^{\prime}}} d^{3} \psi d^{3} \psi^{\prime} .
\end{aligned}
$$

The Fourier components of the Green function can be expressed via integrals of products of the Bessel functions. The corresponding expression for an arbitrary aspect ratio $\sigma_{y} / \sigma_{x}$ and a finite crossing angle in the horizontal plane (but without account of the bunch length effects, $\varphi=\chi=0$ ) was given in Ref.[4]. Here we will derive the expression including the bunch length effects in the most easily tractable case of horizontal oscillations $\left(m_{y}=0\right)$ in horizontally flat beams $\left(\sigma_{x} \gg \sigma_{y}\right)$.

Making use of the identity

$$
\ln \left(x^{2}+y^{2}\right)=-\int_{-\infty}^{\infty} e^{i k x-|k y|} \frac{d k}{|k|}+\text { const }
$$

and performing integration over the betatron phase angles we get 


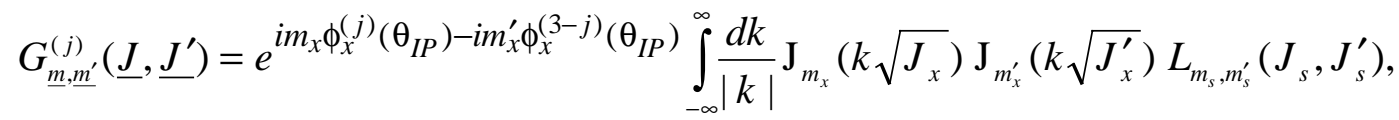

$$
\begin{aligned}
& L_{m_{s}, m_{s}^{\prime}}\left(J_{s}, J_{s}^{\prime}\right)=\frac{1}{(2 \pi)^{2}} \iint d \psi_{s} d \psi_{s}^{\prime} e^{-i m_{s} \psi_{s}+i m_{s}^{\prime} \psi_{s}^{\prime}-i\left(m_{x}+m_{x}^{\prime}\right) \varphi-i \chi\left(m_{x} \sigma-m_{x}^{\prime} \sigma^{\prime}\right)} \times \\
& \exp \left[\frac{i(-1)^{j-1} k d_{x}-\left|k d_{y}\right|+i \alpha k\left(\sigma-\sigma^{\prime}\right)}{\sigma_{x} \sqrt{2\left(1+b^{2}\right)}}\right],
\end{aligned}
$$

Neglecting second order effects in $\sigma_{s} / \beta_{x}{ }^{*}$ we can perform the integration over the synchrotron phase angles as well:

$$
\begin{gathered}
L_{m_{s}, m_{s}^{\prime}}\left(J_{s}, J_{s}^{\prime}\right)=\exp \left[\frac{i(-1)^{j-1} k d_{x}-\left|k d_{y}\right|}{\sigma_{x} \sqrt{2}}\right] \mathrm{J}_{m_{s}}\left[\left(\omega k-\sqrt{2} m_{x} \chi \sigma_{s}+\frac{m_{x}+m_{x}^{\prime}}{\sqrt{2} \beta_{x}^{*}} \sigma_{s}\right) \sqrt{J_{s}}\right] \times \\
\mathbf{J}_{m_{s}^{\prime}}\left[\left(\omega k-\sqrt{2} m_{x}^{\prime} \chi \sigma_{s}+\frac{m_{x}+m_{x}^{\prime}}{\sqrt{2} \beta_{x}^{*}} \sigma_{s}\right) \sqrt{J_{s}^{\prime}}\right],
\end{gathered}
$$

where

$$
\omega=\alpha \sigma_{s} / \sigma_{x}
$$

is (half) the reduced crossing angle.

\section{$5 \quad$ Spectrum of uncoupled modes}

Discarding for a moment the second term in the r.h.s. of eq.(4.12) we can write for the solution of this equation

$$
f_{\underline{m}}^{(k)} \sim \exp \left(-i \underline{m} \cdot \underline{v}^{(k)} \theta\right) .
$$

The beam-beam force contains all Fourier harmonics $n$ in $\theta$ and, being nonlinear, can couple a given mode $\underline{m}$ to all $\underline{m}^{\prime}$ such that

$$
\underline{m}^{\prime} \cdot \underline{v}^{(k)} \approx n+\underline{m} \cdot \underline{v}^{(3-k)}, \quad k=1,2 .
$$

In order to neglect coupling let us suppose that the working point is chosen so that condition (5.2) does not hold for any sufficiently low $\underline{m}^{\prime}$ (no exact criterion to be given) and ignore possible complications due to low synchrotron tune. Then we may retain in the coherent part of the r.h.s. of eq.(4.12) only one term with $\underline{m}^{\prime}=\underline{m}$.

\section{General remark}

Retaining just one term with $\underline{m}^{\prime}=\underline{m}$ in the Fourier expansion of the Green function, we effectively replace it with a function of the difference argument, $G_{\underline{m} \underline{m}} \exp \left[\underline{i m} \cdot\left(\underline{\psi}-\underline{\psi^{\prime}}\right)\right]$, so that the integral operators $\hat{G}^{(k)}$ defined by the last of eqs.(4.7) become commutative with the differential operator $\underline{\hat{D}}$ to make the Vlasov operator $\hat{A}$ selfadjoint. As a consequence its spectrum should be real, which means no instability due to beam-beam interaction without mode coupling (i.e. outside resonances).

Now let us extract the integer parts $\underline{n}^{(k)}$ (allowing for unequal values) from the single particle tunes and decompose the fractional parts into the mean $\underline{v}$ and the difference $\pm \underline{\xi} \underline{\Delta}$ (taking out the normalization parameter $\xi$ defined later):

$$
\underline{\mathbf{v}}_{0}^{(k)}=\underline{n}^{(k)}+\underline{v}+(-1)^{k} \xi \underline{\Delta},
$$

and then introduce new slowly varying vector functions 


$$
\mathbf{u}_{\underline{m}}=\left(\begin{array}{cc}
e^{i \underline{m} \cdot\left(\underline{n}^{(1)}+\underline{v}\right) \theta} & 0 \\
0 & e^{i \underline{i m} \cdot\left(\underline{n}^{(2)}+\underline{v}\right) \theta}
\end{array}\right) \cdot \underline{\mathbf{f}}_{\underline{m}}
$$

into the reduced eq.(4.12). Retaining in the Fourier expansion of the $\delta$-function only terms $\exp \left[ \pm i n_{0}\left(\theta-\theta_{I P}\right)\right] / 2 \pi, n_{0} \equiv \underline{m} \cdot\left(\underline{n}^{(1)}-\underline{n}^{(2)}\right)$, which do not vanish on averaging over $\theta$, we obtain

$$
i \frac{\partial}{\partial \theta} \mathbf{u}_{\underline{m}}=\xi \hat{A}_{\underline{m}} \cdot \mathbf{u}_{\underline{m}},
$$

where the reduced Vlasov operator

$$
\hat{A}_{\underline{m}}=\left(\begin{array}{cc}
\underline{m} \cdot\left(-\underline{\Delta}+\underline{v}_{b b}^{(1)} / \xi\right) & 0 \\
0 & \underline{m} \cdot\left(\underline{\Delta}+\underline{v}_{b b}^{(2)} / \xi\right)
\end{array}\right)+\underline{m} \cdot \underline{\varepsilon^{-1}} \sum_{I P} \frac{r_{p} \sqrt{N_{1} N_{2}}}{2 \pi \gamma \xi}\left(\begin{array}{cc}
0 & e^{i n_{0} \theta_{I P}} \hat{G}_{\underline{m} \underline{m}}^{(1)} \\
e^{-i n_{0} \theta_{I P}} \hat{G}_{\underline{m} \underline{m}}^{(2)} & 0
\end{array}\right)
$$

is self-adjoint by virtue of eqs.(4.10) and (4.13).

Following Yokoya et al. [1] we will solve eq.(5.5) by expansion in the eigenfunctions of the operator $\hat{A}_{\underline{m}}$ :

$$
\hat{A}_{\underline{m}} \cdot \Psi_{\lambda}=\lambda \Psi_{\lambda} .
$$

As noted earlier the eigenvalues $\lambda$ are real. The question remains whether there exist discrete spectral lines (not Landau damped by the beam-beam tunespread) which may go unstable due to interaction with the external impedances. The main interest in this respect is presented by the dipole modes, for definiteness we will consider the horizontal one which is described by the terms with $\underline{m}=( \pm 1,0,0)$ in expansion (4.11). Then it is natural to take for $\xi$ the horizontal beam-beam parameter (negative in the $p-p$ case) for the first (weak) beam

$$
\xi=-\frac{r_{p} N_{2} \beta_{x}^{*}}{2 \pi \gamma \sigma_{x e f f}\left(\sigma_{x e f f}+\sigma_{y}\right)}=-\frac{r_{p} N_{2}}{2 \pi \gamma \varepsilon_{x}\left(1+\omega^{2}\right)\left(1+r_{\sigma}\right)},
$$

where $\sigma_{x \text { eff }}=\sigma_{x}\left(1+\omega^{2}\right)^{1 / 2}, r_{\sigma}=\sigma_{y} / \sigma_{x \text { eff }}, \omega$ being given by eq.(4.16).

\subsection{Spectral density of dipole oscillations}

Introducing the scalar product of two vector functions

$$
(\mathbf{f}, \mathbf{g}) \equiv\left(f^{(1)}, g^{(1)}\right)+\left(f^{(2)}, g^{(2)}\right)=\int\left(\bar{f}^{(1)} g^{(1)}+\bar{f}^{(2)} g^{(2)}\right) d^{3} J,
$$

where the bar means complex conjugation, we can normalize the eigenfunctions $\boldsymbol{\Psi}_{\lambda_{n}}$ (the index $n$ makes provision for possible degeneracy of eigenvalues) in such a way that

$$
\left(\boldsymbol{\Psi}_{\lambda m}, \boldsymbol{\Psi}_{\mu n}\right)=\delta_{\lambda \mu} \boldsymbol{\delta}_{m n},
$$

where $\delta_{\lambda \mu}$ is the Kronecker symbol if $\lambda$ belongs to the discrete spectrum, $\boldsymbol{P}$, and the Dirac $\delta$ function if $\lambda$ belongs to the continuous spectrum, $\boldsymbol{C}$. If $\boldsymbol{P}$ is not empty, then integrals over the spectrum are the Stieltjes integrals performed with the help of the integrating function $w(\lambda)$ with the following properties

$$
\begin{aligned}
& w(\lambda+0)-w(\lambda-0)=1, \quad \lambda \in \boldsymbol{P}, \\
& d w(\lambda) / d \lambda=1, \quad \lambda \in \boldsymbol{C}, \\
& d w(\lambda) / d \lambda=0, \quad \lambda \notin \boldsymbol{C}, \boldsymbol{P} .
\end{aligned}
$$

Important characteristics of the dipole eigenmodes are the coefficients 


$$
c_{n}^{(k)}(\lambda)=\left(\Psi_{0}, \Psi_{\lambda n}^{(k)}\right), \quad k=1,2
$$

where

$$
\Psi_{0}=\sqrt{J_{x}} e^{-\left(J_{x}+J_{y}+J_{s}\right) / 2},
$$

which give (the first one after multiplication by $r_{\xi}^{1 / 2}$ ) the relative amplitude of the eigenmode excitation by a dipole kick delivered to the corresponding beam. They also give (the first one after multiplication by $r_{\xi}^{-1 / 2}$ ) the amplitude of the center-of-mass oscillation of the beams when the corresponding eigenmode is excited with unit amplitude.

The spectral density of the center-of-mass oscillations observed in beam $k$ after a dipole kick at beam $j$ can be expressed via these coefficients and the integrating function as

$$
s_{k j}(\lambda)=r_{\xi}^{(k-j) / 2} \sum_{n} c_{n}^{(k)}(\lambda) c_{n}^{(j)}(\lambda) \frac{d w(\lambda)}{d \lambda}
$$

In what follows we will present the spectral density by histograms obtained by integrating (5.14) over small intervals $(\Delta \lambda=0.025 \div 0.05)$.

In the presence of external impedances (assumed identical for both beams) the induced (complex) tune shift (assumed small w.r.t. $\xi$ ) for a mode with discrete eigenvalue $\lambda_{0} \in \boldsymbol{P}$ is equal to the tune shift which the strong beam would experience alone times the factor

$$
r_{b b}=r_{\xi}\left[c^{(1)}\left(\lambda_{0}\right)\right]^{2}+\left[c^{(2)}\left(\lambda_{0}\right)\right]^{2} .
$$

This factor can be quite small reducing the instability growth rate (if any) to a negligible value even in the absence of Landau damping.

\subsection{Effect of tune split and unequal intensities}

Let us introduce the Yokoya factor, which is the maximum discrete eigenvalue (in units of $\xi$ )

$$
Y=\max \{\lambda\},
$$

and remind the results which pertain to the simplest case of a single IP, equal intensities and tunes [1]. Due to symmetry between the beams the range of definition of operator (6) splits into the direct sum of two invariant subspaces corresponding to $\Sigma$-modes $f^{(1)}=f^{(2)}=f^{(+)}$ and $\pi$-modes $f^{(1)}=-f^{(2)}=f^{(-)}$. The spectrum of each family comprises the continuum, $\boldsymbol{C}=(0,1)$, and a number of discrete eigenvalues. The $\Sigma$-modes of dipole oscillations $(m=1)$ have one discrete eigenvalue, $\lambda=0$, the corresponding eigenfunction (multiplied by $2^{1 / 2}$ ) coincides with $\Psi_{0}$ given by eq.(13). The maximum discrete eigenvalue of the dipole $\pi$-modes appears to depend on the aspect ratio $r_{\sigma}$ : it is $Y=1.214$ for round beams $\left(r_{\sigma}=1\right)$ and $Y=1.330$ for flat beams $\left(r_{\sigma} \ll 1\right)$. The number of discrete eigenvalues also depends on the aspect ratio, for flat beams there are two more discrete eigenvalues [4]: $\lambda_{2}=1.026, \lambda_{3}=1.002$. However, they are physically insignificant since their spectral weight (15) is small (0.035 and 0.003 compared to 0.524 for the first eigenvalue).

Breaking the symmetry between the beams weakens the coherence of their oscillations reducing the Yokoya factor, $Y$. With decreasing intensity ratio, $r \xi$, the largest discrete eigenvalue merges with the continuum at $r_{\xi} \approx 0.6$ [2]. However, the discrete mode with $\lambda=0$ survives. Fig.2 (left column) shows the spectral density seen in the weak beam, $s_{11}$, at $r_{\xi}=1$, 0.65 and 0.3 (round beams case). It is interesting to note that even at $r \xi=0.3$ the spectrum has a pronounced peak at $\lambda \approx 0.8$ (in $s_{22}$ it can also be seen but is less sharp).

An efficient method to damp both discrete modes is splitting the tunes in the two beams as proposed in Ref.[3]. It was shown that for flat beams a half split $|\Delta| \approx 0.75$ (in units of $\xi$ ) is needed for the damping [4]. Approximately the same tune split is necessary in the case of round beams as well. 

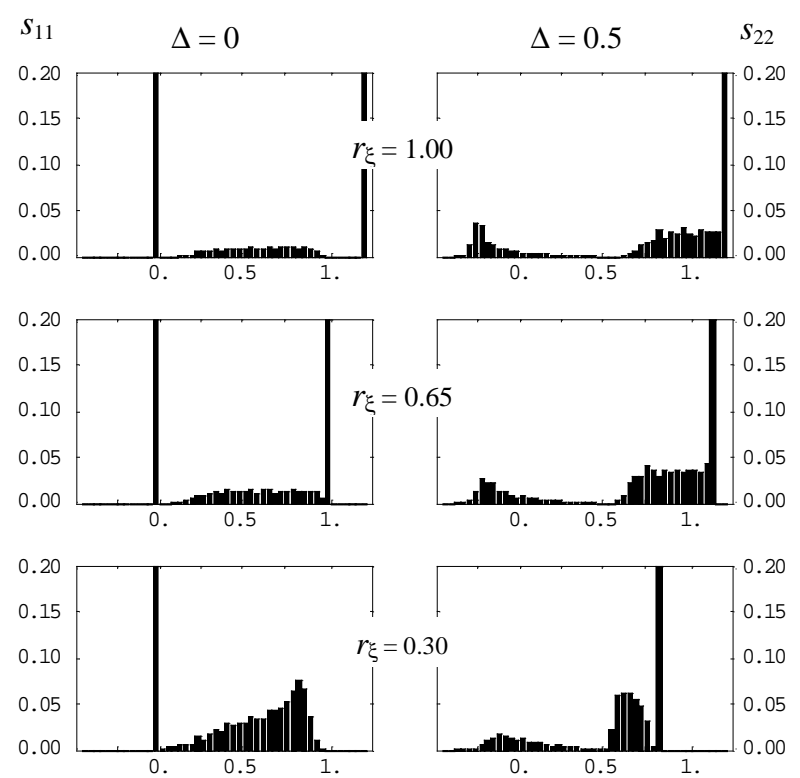

Figure 2. Spectral density of dipole oscillations $s_{k k}(\lambda)$ : left column - in the weak beam in the case of no tunesplit; right column - in the strong beam in the case of tunesplit $2 \Delta=1$ at indicated values of the intensity ratio.

Fig.2 (top right) shows the spectral density $s_{22}$ of dipole oscillations in round beams of equal intensities at $\Delta=0.5$. The largest discrete eigenvalue can be seen on the verge of merging with the continuum.

However, in the case of unequal intensities the effect of the tune split depends on the sign of $\Delta$. If the tune split counteracts the beam-beam tuneshift for the strong beam $(\Delta<0)$ then even smaller absolute values of the tune split, $|\Delta|$, are necessary for the damping. In the opposite case $(\Delta>0)$ the discrete mode can reappear even if it had been damped due to unequal intensities (see Fig.2, bottom right).

Due to this interference of different damping mechanisms it seems safer to make the tune split even larger than necessary in the case of equal intensities, e.g. by placing the working points for the two beams in different cells in the tune diagram. However, in such a case some coherent beam-beam resonances of relatively low order can be encountered, as will be discussed in Section 7.

\subsection{Effect of redistribution of phase advances}

Some new possibilities to damp the coherent modes arise if a given pair of bunches collide at two IPs, whose position without loss of generality may be assumed to be $\theta_{I P}=0, \pi$. If the IPs are identical (equal optical functions, crossing angles, offsets) then there are two methods to achieve complete cancellation of the effect of coherent interaction at these IPs.

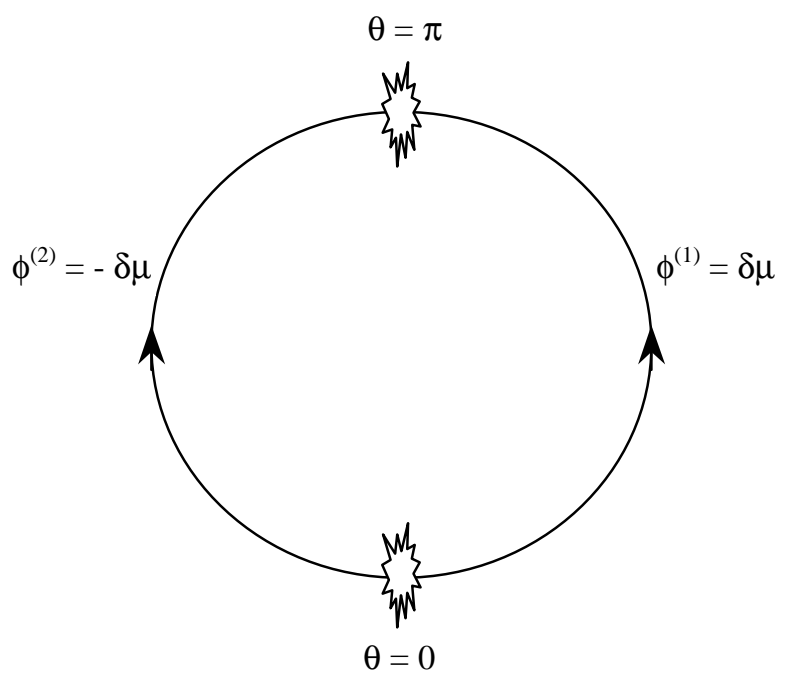

Figure 3. Scheme of phase advance redistribution between two interaction points.

The first method can be implemented in machines with separate rings for the two beams. Its idea is obvious from the form of the second term in the r.h.s. of eq.(5.6). If the integer tune split $n_{0}$ is an odd number then contributions from the two IPs to the sum have opposite signs, annihilating each other. In the result only the incoherent part of operator $\hat{A}_{\underline{m}}$ remains, which produces purely continuous spectrum.

Another method involves phase advance redistribution between the two IPs and is applicable in one ring machines as well. Normally $\theta=\pi$ is the symmetry point: $\left.\underline{\mu}\right|_{\theta=\pi}=\pi \underline{v}$ so that $\left.\underline{\phi}\right|_{\theta=\pi}=0$ (let us note that $\left.\phi\right|_{\theta=0}=0$ by definition). It is possible, however, to break this symmetry by redistributing the betatron phase advance as shown in Fig. 3 without changing the total value. Assuming the one ring case we have 


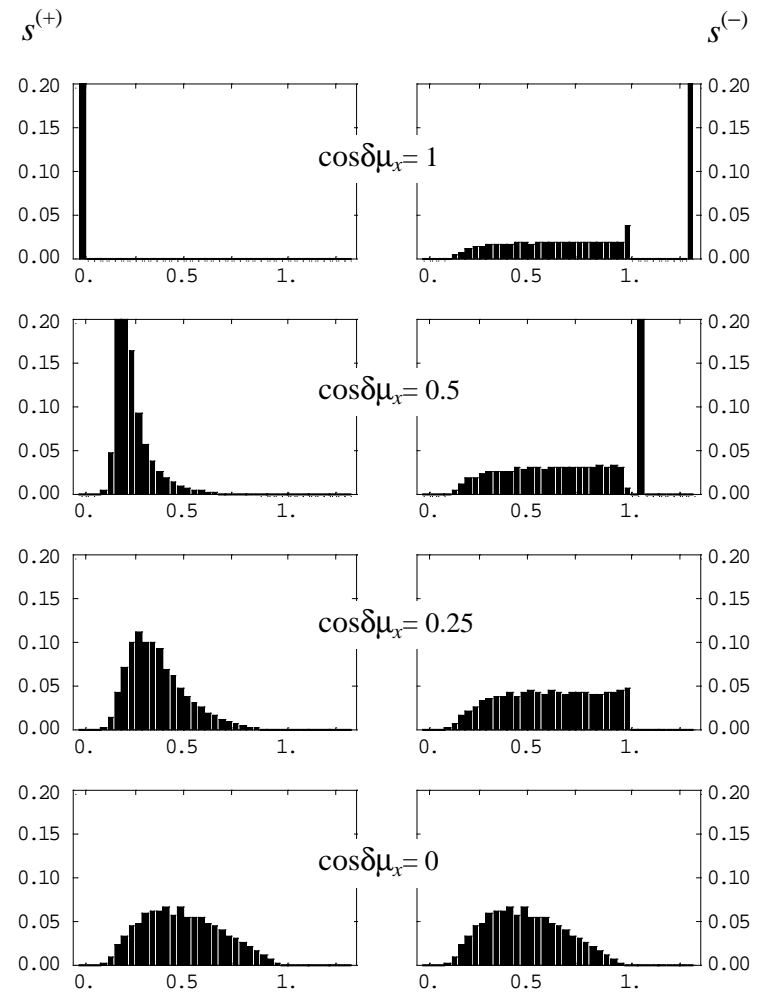

Figure 4. Spectra of quasi $\Sigma$-modes (left) and quasi $\pi$-modes (right) at increasing (from top to bottom) values of $\delta \mu_{x}$.

$$
\left.\phi_{x}^{(1)}\right|_{\theta=\pi}=-\left.\phi_{x}^{(2)}\right|_{\theta=\pi}=\delta \mu_{x} .
$$

As can be seen from expression (4.15), the Green function for dipole oscillations $\left(m_{x}=m_{x}{ }^{\prime}=1\right)$ has the exponential factor which equals unity at $\theta_{I P}=0$ and

$$
e^{ \pm i m_{x}\left(\phi_{x}^{(1)}-\phi_{x}^{(2)}\right)}=e^{ \pm 2 i \delta \mu_{x}}
$$

at $\theta_{I P}=\pi$. If $\delta \mu_{x}=\pi / 2$ then again the contributions from the two IPs annihilate each other.

It is worthwhile to see whether such a large variation in the phase advance is really necessary. Let us consider an arbitrary $\delta \mu_{x}$ and limit ourselves to the case of no tunesplit, no offsets nor crossing angle, of negligible bunch length and equal intensities in flat beams. Then we have from eq.(4.15) for the Green function diagonal elements (extending the analysis on arbitrary $m_{x}=m_{x}{ }^{\prime}=m>0$, $m_{s}=m_{s}^{\prime}=0$ )

$$
\begin{aligned}
& G_{\underline{m}, \underline{\underline{m}}}^{(k)}\left(\underline{J}, \underline{J^{\prime}}\right)=\exp \left[2 i m \phi_{x}^{(k)}\left(\theta_{I P}\right)\right] G_{m}\left(J_{x}, J_{x}^{\prime}\right), \\
& G_{m}\left(J_{x}, J_{x}^{\prime}\right) \equiv \frac{1}{m}\left(\frac{\min \left(J_{x}, J_{x}^{\prime}\right)}{\max \left(J_{x}, J_{x}^{\prime}\right)}\right)^{m / 2}
\end{aligned}
$$

The reduced Vlasov operator with regard of eq.(5.17) has the form

where

$$
\hat{A}_{\underline{m}}=m\left(\begin{array}{cc}
2 Q_{x} & -\left(1+e^{2 i m \delta \mu_{x}}\right) \hat{G}_{m} \\
-\left(1+e^{-2 i m \delta \mu_{x}}\right) \hat{G}_{m} & 2 Q_{x}
\end{array}\right),
$$

$$
Q_{x}(\underline{J})=\left(1-e^{-J_{x}}\right) / J_{x}
$$

is the normalized incoherent tuneshift per IP, the operator $\hat{G}_{m}$ is associated with its kernel as shown in the first of eqs.(4.13).

The matrix operator (5.20) can be rendered real (and symmetric in symbolic form) by one more transformation

$$
\mathbf{v}_{m}=\left(\begin{array}{cc}
e^{-i m \delta \mu_{x} / 2} & 0 \\
0 & e^{i m \delta \mu_{x} / 2}
\end{array}\right) \cdot \mathbf{u}_{\underline{m}} .
$$

Due to the symmetry, the eigenmodes again split into two families: quasi $\Sigma$-modes: $v_{m}^{(1)}=v_{m}^{(2)} \equiv v_{m}^{(+)}$, and quasi $\pi$-modes: $v_{m}^{(1)}=-v_{m}^{(2)} \equiv v_{m}^{(-)}$, for which we obtain decoupled eigenvalue problems:

$$
\frac{\lambda}{m} v_{m}^{( \pm)}=\left(Q_{x} \mp \cos \delta \mu_{x} \hat{G}_{m}\right) v_{m}^{( \pm)} .
$$


Fig. 4 shows the evolution of the spectrum of the dipole oscillations $(m=1)$ with increasing $\delta \mu_{x}$. As the contribution of the integral operator to the r.h.s. of eq.(5.23) decreases, the discrete eigenvalues merge with the continuum. At $\delta \mu_{x} \approx 75^{\circ}$ only the continuous spectrum is left for both quasi $\pi$ - and $\Sigma$-modes.

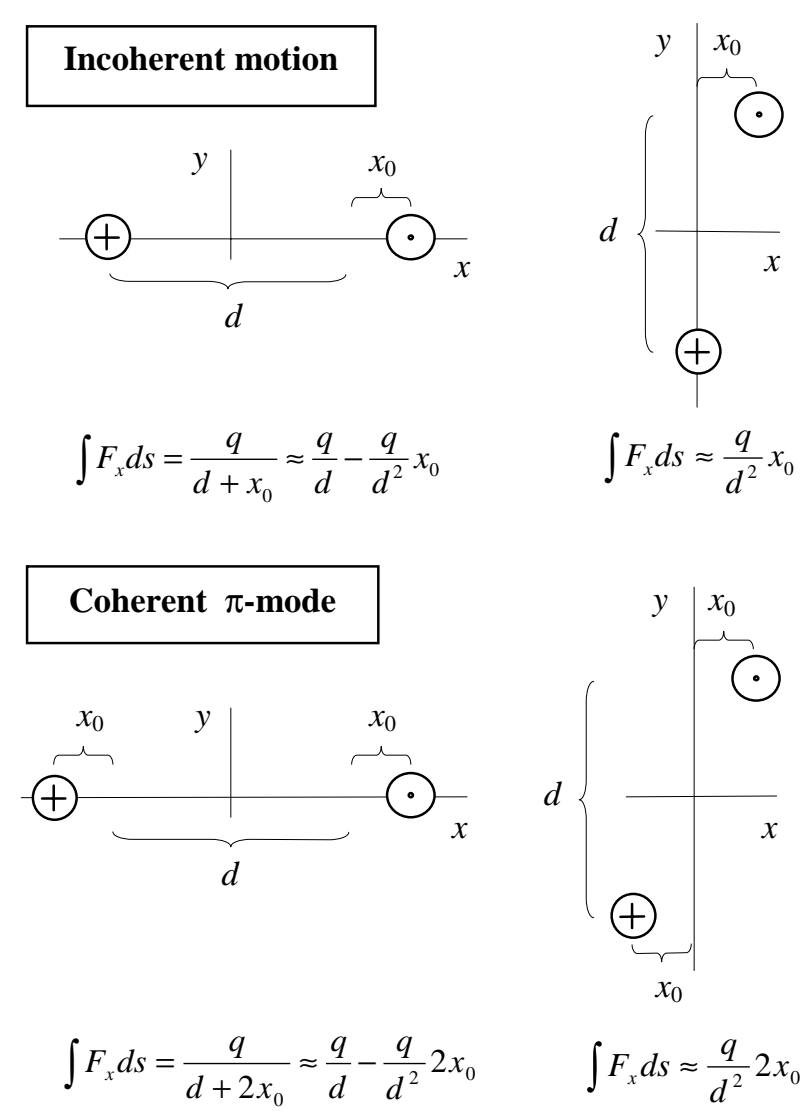

Figure 5. Schematic picture of incoherent and coherent effects of long-range interactions of pencil beams; $q=2 e^{2} N / \gamma$.

\section{Long-range interactions}

So far we considered head-on interactions of just two bunches at one or two identical IPs. In LHC each bunch will experience numerous parasitic long-range interactions [11], the particular number (more than 100 including those in IP2 and IP8) depends on the bunch position in the train.

An important feature of the longrange interactions is their relatively large contribution to the coherent tune shift as illustrated with Fig.5. Simple considerations show that the Yokoya factor for long-range interactions alone would be $Y=2$ with both horizontal and vertical separation. The tune shift has the opposite sign of that from the head-on interactions, if the beams are separated in the plane of oscillations (horizontal in our case), and the same sign if separation takes place in the other (vertical) plane. One may expect that a sufficient number $N_{\text {LR }}$ of long-range interactions in the first case can compensate for the head-on tune shift, bringing the horizontal coherent tune within the incoherent tune spread. With $N_{\text {LR }}$ increasing further the coherent line

should reappear on the other side of the range of incoherent tunes.

To consider the effect of multiple interactions we have to generalize the basic formulae of Sections 4 and 5.

\subsection{Multi-bunch modes}

To simplify the problem we will ignore the gaps between the bunch trains and assume bunches in both beams to be distributed equidistantly over the machine circumference. Let us numerate consecutively all possible interaction points ( $2 K$ for $K$ bunches/beam) starting from, say, IP1. Then $\theta_{I P}=\pi(I P-1) / K$. Nonexistent IPs can be discarded with the help of the parameter $\eta_{I P}$ equal to 1 for actual IPs and zero otherwise.

Now we should mark a particular bunch with a double index, let $\{k, \beta\}$ mean the $\beta$-th bunch of the $k$-th beam. The index of its conjugate (i.e. the bunch it encounters) at a given IP can be written as

$$
\overline{\{k, \beta\}}_{I P}=\left\{3-k, 1+\operatorname{Mod}\left[\beta-1-(-1)^{k}(I P-1), K\right]\right\},
$$

where $\operatorname{Mod}[n, m] \in(0, m-1)$ is the non-negative remainder on division of $n$ by $m$.

The multi-bunch normal modes can be introduced by the relations [1] 


$$
g_{n}^{(k)}=\frac{1}{K} \sum_{\beta=1}^{K} e^{-2 \pi i n \beta / K} f^{\{k, \beta\}}, \quad n=0, \ldots, K-1
$$

The inverse transformation gives the distribution function of individual bunches:

$$
f^{\{k, \beta\}}=\sum_{n=0}^{K-1} e^{2 \pi i n \beta / K} g_{n}^{(k)}, \quad \beta=1, \ldots, K .
$$

Applying transformation (6.2) to the Vlasov equations (4.4) we obtain a system of independent equations of the form (4.6) for vectors

$$
\mathbf{g}_{n}=\left(\begin{array}{l}
g_{n}^{(1)} \\
g_{n}^{(2)}
\end{array}\right)
$$

the corresponding Vlasov operator being

$$
\hat{A}_{n}=\left(\begin{array}{cc}
\underline{v}^{(1)} \cdot \underline{\hat{D}} & 0 \\
0 & \underline{v}^{(2)} \cdot \underline{\hat{D}}
\end{array}\right)+\underline{\varepsilon^{-1}} \cdot \underline{\hat{D}} \sum_{I P=1}^{2 K} \eta_{I P} \frac{r_{p}}{\gamma} \sqrt{N_{1} N_{2}} \delta_{\mathrm{p}}\left(\theta-\theta_{I P}\right)\left(\begin{array}{cc}
0 & e^{2 n i \theta_{I P}} \hat{G}^{(1)} \\
e^{-2 n i \theta_{I P}} \hat{G}^{(2)} & 0
\end{array}\right)
$$

\section{General remark:}

The second matrix operator in the r.h.s. of eq.(6.5) is self-adjoint for all $n$, therefore the presence of many bunches per beam can not by itself lead to instability (as long as the equilibrium state exists), the only source of instability is still the coupling of different Fourier harmonics of the distribution function in the phase angles.

Let us continue with an uncoupled angular mode $\underline{m}$ denoting the corresponding Vlasov operator as $\hat{A}_{n, \underline{m}}$. There is an additional exponential phase factor in its coherent part coming from the Green function (see eq.(4.15)). However, if all actual interaction points have regular betatron phase advances,

$$
\eta_{I P} \underline{\phi}^{(k)}\left(\theta_{I P}\right)=0
$$

then they contribute in phase into the coherent part of $\hat{A}_{n, \underline{m}}$ for some $n$ (for $n=0$ at least). For such $n$ the spectrum of operator $\hat{A}_{n, \underline{m}}$ has the largest span and the maximum number of discrete eigenvalues (for a given $\underline{m}$ ). For other $n$, called intermediate modes, the contributions from different interaction points into the coherent part cancel each other (partially or completely) leaving in the result only the continuous spectra.

For parasitic interaction points close to the nominal IPs, condition (6.6) does not hold since

$$
\phi_{u}^{(1)}(\theta) \equiv \mu_{u}^{(1)}(\theta)-v_{u}^{(1)} \cdot \theta \approx \arctan \frac{R \theta}{\beta_{u}^{*}}, \quad \phi_{u}^{(2)}(\theta)=-\phi_{u}^{(1)}(\theta), \quad u=x, y,
$$

where we have chosen the position of the nominal IP for $\theta=0$. Assuming $\underline{\phi}^{(1)}\left(\theta_{I P}\right)=-\underline{\phi}^{(2)}\left(\theta_{I P}\right)=\underline{\phi}_{I P}$ everywhere (as in a one-ring machine) we will obtain the total exponential phase factors in the coherent part of $\hat{A}_{n, \underline{m}}$

$$
\exp \left[ \pm 2 i\left(n \theta_{I P}+\underline{m} \cdot \underline{\phi}_{I P}\right)\right] .
$$

Though $\phi_{I P}$ is not a linear function of $\theta_{I P}$ still there is a possibility that for some $n$ the majority of interaction points contribute (almost) in phase. Let us assume that such $n$ exists and lump all long-range and head-on interactions together setting all phase factors (6.8) equal unity. In this way we will obtain an upper bound on the effect of the long-range interactions. 

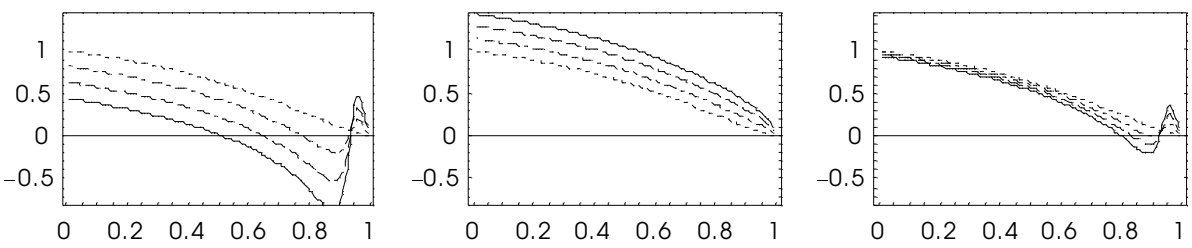

Figure 6. Normalized incoherent tune shift $v_{x b b} / \xi_{x}^{\text {(head-on) }}$ vs. $J_{x} /\left(1+J_{x}\right)$ for one head-on and $N_{\mathrm{LR}}=0,4,8,12$ long-range interactions (indicated with increasing solidity of the line) at $d=5 \sigma_{x}$ separation in the case of horizontal (left), vertical (center) and alternating (right) crossing.
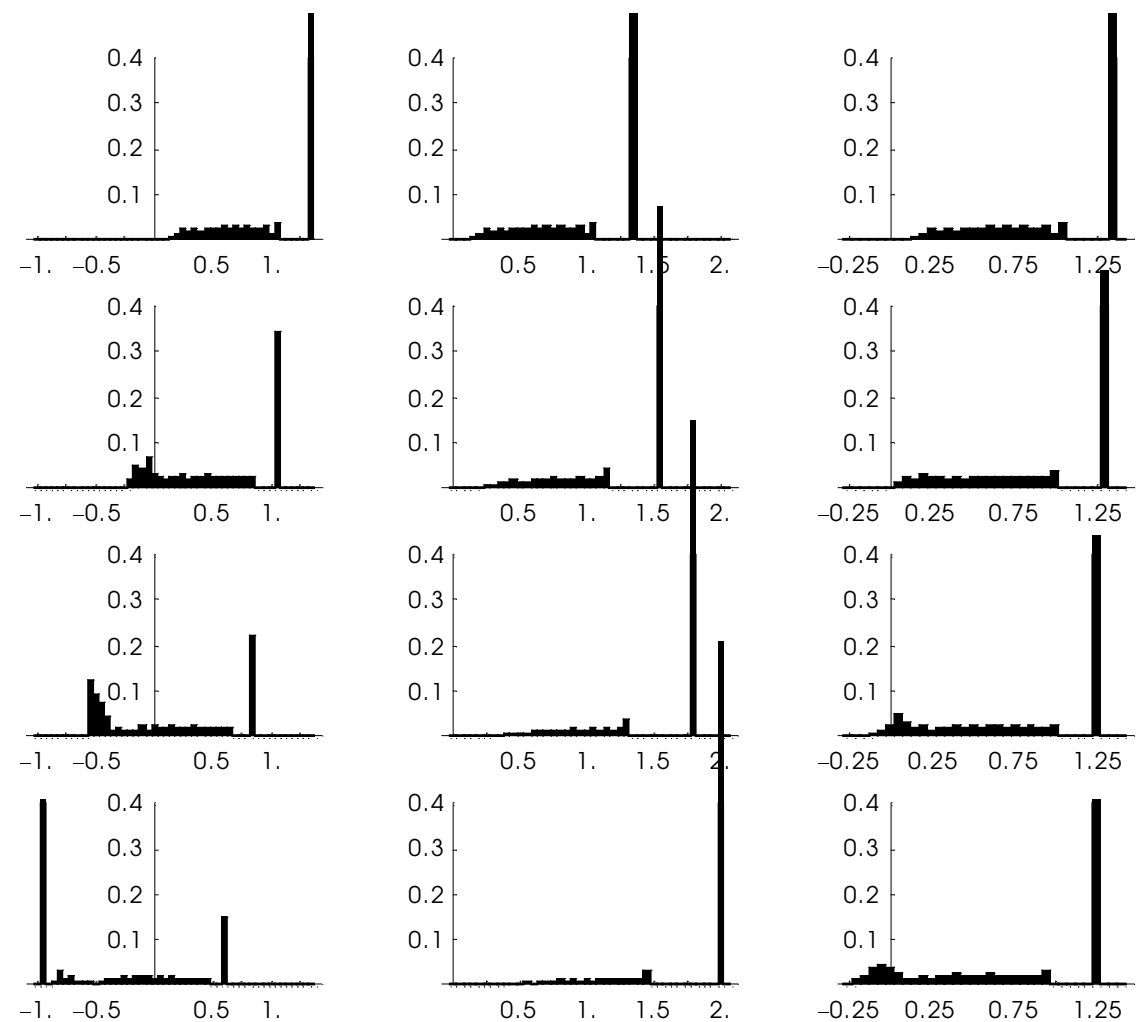

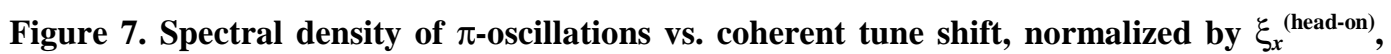
for one head-on and (from top to bottom) $N_{\mathrm{LR}}=0,4,8,12$ long-range interactions in the same cases of separation as in Fig. 6.

\subsection{Effect of lumped long-range interactions}

A further simplification comes from the fact that at the locations of long-range interactions the beta-functions are large rendering negligible the effects of the finite bunch length and crossing angle. In the present section we ignore these effects for both long-range and head-on interactions and consider the case of flat beams with equal tunes and intensities.

To make some features more visible we take a moderate separation of $5 \sigma_{x}$ at the parasitic IPs, thus only 8 interactions are needed to produce the same tune shift as $\sim 30$ longrange interactions at $9.5 \sigma_{x}$ separation, as foreseen in $\operatorname{LHC}[11,12]$. Fig. 6 shows the incoherent horizontal tune shift (normalized by $\xi_{x}^{\text {(head-on) }}$ ) for one head-on and $N_{\mathrm{LR}}=0,4,8,12$ long-range interactions at horizontal (left), vertical (center) and alternating (right) separation. The spectra of horizontal $\pi$-oscillations obtained in these cases are presented in Fig.7.

In the case of horizontal separation the spectrum behaviour with increasing $N_{\mathrm{LR}}$ is more complicated than predicted on the basis of the rigid bunch model. Instead of sweeping across the incoherent tunespread (and being damped while within it) the original discrete line 
stays to the right of the continuum range (see Fig.7, left column). At the same time another line appears on the left side so that there are two discrete $\pi$-mode lines in a situation when one might expect no such lines at all.

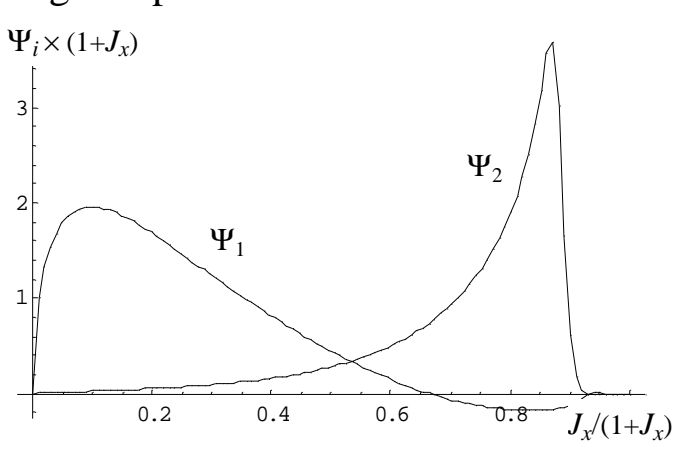

Figure 8. Two discrete $\pi$-mode eigenfunctions at $N_{\mathrm{LR}}=12$; the corresponding eigenvalues being $\lambda_{1}=0.613, \lambda_{2}=-0.928$.
The corresponding eigenfunctions at $N_{\mathrm{LR}}=12$ (multiplied by the square root of the Jacobian of the transformation to variable $\left.u=J_{x} /\left(1+J_{x}\right)\right)$ are shown in Fig.8. The second mode involves mainly tail particles with large $J_{x}$ whose incoherent tune shifts are negative.

On the contrary, in the first mode mainly the core particles participate. From comparison with Fig. 13 one can deduce that with increasing $N_{\mathrm{LR}}$ the range of participating particles is contracted to smaller $J_{x}$ but their perturbation amplitude (given by the eigenfunction value) grows. In the result the relative influence of the head-on interaction on the core particles increases upkeeping the mode tune at the expense of coherence of oscillations described by the coefficient (5.15).

This coefficient (given by the height of the line in the spectrum diagram) is rather small for both discrete lines at $N_{\mathrm{LR}}=8$ rendering more than a 4-fold reduction in the instability growth rate w.r.t. the single beam case.

The situation is different with separation in the other plane (vertical in our case). As can be seen in Fig.7, central column, the $\pi$-mode spectral weight is rapidly increasing with $N_{\text {LR }}$. For vertical oscillations such an increase takes place at horizontal separation. This means that if the beams are separated in one plane at all parasitic IPs, then the coherent stability in the other plane may be deteriorated.

This problem can be solved by implementing the alternating separation proposed in Ref.[13] (in the vertical plane around IP1 and in the horizontal plane around IP5 or vice versa). Fig.7 (right column) shows even a slight reduction in the horizontal $\pi$-mode spectral weight. The same is true for the vertical $\pi$-mode since both planes are equal in this case.

However there is a conflict between the effects of alternating separation and integer tune split (or phase advance redistribution) on the long-range contribution to coherent tune shift. To answer what is the net effect of a simultaneous implementation of these methods, calculations with the actual distribution of phase advances $\phi_{I P}$ should be carried out.

\section{Coherent beam-beam resonances}

Several working points are considered for the LHC operation such that particles can encounter incoherent resonances only of order 13 or higher [11]. Since the coherent tuneshifts of high order modes do not exceed the incoherent ones, the coherent beam-beam resonances in the case of equal single particle tunes are possible only inside stopbands of the corresponding incoherent resonances and therefore present no new danger.

The situation is different if the fractional tunes of the two beams are placed in different cells of the tune diagram in order to suppress the coherent dipole modes. Then the resonance condition (5.2) involving tunes of both beams can be satisfied for a quite low resonance order $m=\left|m_{x}\right|+\left|m_{y}\right|+\left|m_{x}^{\prime}\right|+\left|m_{y}^{\prime}\right|$. With the horizontal tune values considered for LHC [11]

$$
v_{x 1}=0.232, v_{x 2}=0.310, v_{x 3}=0.385
$$

the following resonances can be encountered

$$
3 \mathrm{v}_{x 1}+\mathrm{v}_{x 2}=1.006 ; \quad 2 \mathrm{v}_{x 2}+\mathrm{v}_{x 3}=1.005 ; \quad \mathrm{v}_{x 1}+2 \mathrm{v}_{x 3}=1.002
$$


Let us consider in more detail resonances of the type ${ }^{3}$

$$
m_{1} v_{x}^{(1)}+m_{2} v_{x}^{(2)}=n_{r}
$$

with $m_{y 1}=m_{y 2}=m_{s 1}=m_{s 2}=0$ in the case $\left|v_{x}^{(2)}-v_{x}^{(1)}\right| \gg>|\xi|$. Then we may retain just two components of the distribution functions in eqs.(4.11) combining them in the vector

$$
\mathbf{v}=\left(\begin{array}{c}
f_{m_{1}}^{(1)} \\
e^{-i n_{r} \theta} f_{-m_{2}}^{(2)}
\end{array}\right)
$$

Assuming $\underline{\phi}^{(1)}\left(\theta_{I P}\right)=-\underline{\phi}^{(2)}\left(\theta_{I P}\right)=\underline{\phi}_{I P}$ and extracting the corresponding exponential from the Green function (4.14), we obtain the following equation

$$
i \frac{\partial}{\partial \theta} \mathbf{v}=\left(\begin{array}{cc}
m_{1} v_{x}^{(1)} & m_{1} \sqrt{r_{\xi}} \sum_{I P} \xi_{e} i n_{r} \theta_{I P}+i m \phi_{x I P} \tilde{G}_{m_{1},-m_{2}}^{(1)} \\
-m_{2} \sqrt{r_{\xi}} \sum_{I P} \xi e^{-i n_{r} \theta_{I P}-i m \phi_{x I P}} \tilde{G}_{-m_{2}, m_{1}}^{(2)} & n_{r}-m_{2} v_{x}^{(2)}
\end{array}\right) \cdot \mathbf{v}
$$

where $m=m_{1}+m_{2}$. For odd $m$ the coupling terms are zero unless there is an offset $d_{x} \neq 0$.

Dividing the $j$-th component of the vector $\mathbf{v}$ by $\left|m_{j}\right|$ we can render the matrix operator in the r.h.s. of eq.(7.5) Hermitian if $m_{1} \cdot m_{2}<0$ (difference resonance) or anti-Hermitian if $m_{1} \cdot m_{2}>0$ (sum resonance). In the latter case it may have complex eigenvalues which means instability.

Due to exponential factors at the integral operators, in the case of two IPs there is a possibility of cancellation of their contribution to the excitation of even-order resonances in the absence of phase advance errors, $\phi_{x I P}$. To obtain an upper bound of the growth rate let us allow for the errors to be large enough for constructive contribution from the IPs and neglect the finite bunch length effect.

In the case of flat beams without crossing angle the Green function for even-order resonances $m \geq 4$ and $m_{1}=1$ takes on a simple form,

$$
G_{1,1-m}\left(J_{x}, J_{x}^{\prime}\right)=\left\{\begin{array}{cc}
0, & J_{x}^{\prime}<J_{x}, \\
\sqrt{1-z} \mathrm{P}_{m / 2-1}^{-1}(1-2 z), & z=J_{x} / J_{x}^{\prime}<1,
\end{array}\right.
$$

where $\mathrm{P}_{n}^{m}(x)$ is the associated Legendre function.

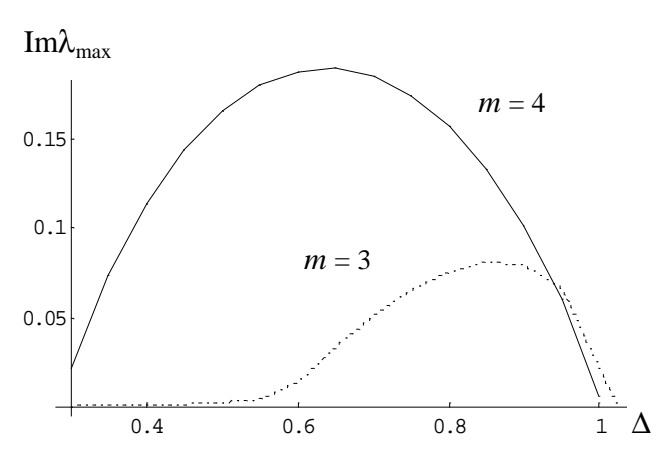

Figure 9. Growth rate on coherent resonances (see text for details).

Fig.9 shows the maximum imaginary part of the eigenvalues (in units of the total incoherent tune shift $\xi^{(\text {tot })}$ ) on $m=4$ resonance (solid line) as a function of the normalized distance

$$
\Delta=\frac{n_{r}-m v_{x 0}}{m \xi^{(\text {tot) }}}
$$

The expression for the $m=3$ resonance Green function in the case of flat beams with small offset was given in Ref.[4]. The results obtained for $d_{x} / \sigma_{x}=0.2$ are shown in Fig. 9 with dotted line.

High growth rates (even with account of partial cancellation of the IPs contributions) on

\footnotetext{
${ }^{3}$ The particular case $m_{1}=m_{2}$ was earlier considered on the basis of the Vlasov equation in Ref.[14].
} 
resonances (2) can create problems in operation with fractional tunes in different cells. Therefore the integer tune split discussed in Section 5.3 is a more viable option.

It should be mentioned that in the round beam case the coherent resonances were studied analytically, with the help of a Mathematica notebook [15] which employs expansion in the Laguerre polynomials, and numerically [16] by tracking an ensemble of particles with the beam-beam force obtained from a Gaussian fit of their distribution. However, the accuracy of both methods for high orders, $m \geq 4$, is questionable since in the first case a very large number of polynomials is necessary whereas in the second case the multipole components of the beam-beam force are not properly represented.

\section{Influence of synchrotron motion on coherent beam-beam modes}

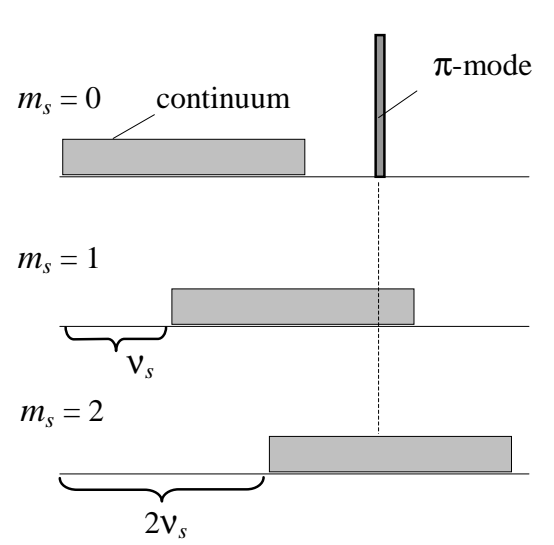

Figure 10. Principle of damping by synchrotron sidebands of the continuum modes.
So far we ignored the synchrotron oscillations which can couple to the transverse motion owing to finite bunch length, crossing angle and/or dispersion function. The synchro-betatron coupling affects the coherent beam-beam modes in two ways. First, an additional degree of freedom weakens the coherence of oscillations reducing the Yokoya factor and the spectral weight of discrete lines. Second, if the synchrotron tune is comparable with the beam-beam parameter, the synchrotron sidebands of the continuum modes can overlap discrete lines, as illustrated by Fig.10, and provide their Landau damping.

A general treatment of Landau damping by the sidebands is given in Appendix A. Here we consider the effects of particular coupling mechanisms in the

case of flat beams with equal tunes and equal intensities colliding without offsets at one IP.

Let us return to the Vlasov equation in the form (4.12) leaving in it only the horizontal dipole mode and its synchrotron satellites of relatively low order $l: \underline{m}(l)=(1,0, l)$, $l=0, \pm 1, \pm 2, \ldots$ which have tunes in the close neighborhood of $v_{x}$, so that only the constant $1 / 2 \pi$ can be retained in the Fourier expansion of the $\delta$-function. Owing to the assumed equality of the beams and absence of offsets we can introduce $\Sigma$ - and $\pi$-modes

$$
u_{l}^{( \pm)}=\frac{1}{2} e^{i v_{x 0} \theta} \cdot\left(f_{\underline{m}(l)}^{(1)} \pm f_{\underline{m}(l)}^{(2)}\right)
$$

and obtain for them independent systems of equations

$$
\frac{i}{\xi} \frac{\partial}{\partial \theta} u_{l}^{( \pm)}=\left[Q_{x}(\underline{J})+l Q_{s}\right] u_{l}^{( \pm)} \mp\left(1+l \varepsilon_{x} / \varepsilon_{s}\right) \sum_{l^{\prime}} \hat{G}_{l, l^{\prime}} u_{l^{\prime}}^{( \pm)},
$$

where $Q_{x}=v_{x \mathrm{bb}} / \xi$ is the normalized beam-beam tuneshift, $Q_{s}=\mathrm{v}_{s} / \xi$, the kernel of the integral operator is given by eqs.(4.15) with the additional factor $\left(1+\omega^{2}\right)$ in the case of finite crossing angle. In the following we will set $\varepsilon_{x} / \varepsilon_{s} \rightarrow 0$ which is justified in all practical cases.

\subsection{Effect of finite bunch length and chromaticity}

If there are neither offsets nor crossing angle the Green function (4.15) can be factorized into a product of transverse and longitudinal parts,

$$
G_{l, l^{\prime}}\left(\underline{J}, \underline{J^{\prime}}\right)=G_{1}\left(J_{x}, J_{x}^{\prime}\right) \cdot L_{l, l^{\prime}}\left(J_{s}, J_{s}^{\prime}\right) \text {, }
$$

where $G_{1}$ is given by eq.(5.19) with $m=1$ and 


$$
\begin{aligned}
L_{l, l^{\prime}}\left(J_{s}, J_{s}^{\prime}\right)= & \frac{1}{(2 \pi)^{2}} \iint d \psi_{s} d \psi_{s}^{\prime} e^{-i l \psi_{s}+i l^{\prime} \psi_{s}^{\prime}-2 i \varphi-i \chi\left(\sigma-\sigma^{\prime}\right)}= \\
& =2 \int_{0}^{\infty} e^{-t} \mathrm{~J}_{l}\left[\left(\frac{t}{2 \beta^{*}}-\chi\right) \sigma_{s} \sqrt{2 J_{s}}\right] \mathrm{J}_{l^{\prime}}\left[\left(\frac{t}{2 \beta^{*}}-\chi\right) \sigma_{s} \sqrt{2 J_{s}^{\prime}}\right] d t- \\
& -(-1)^{l+l^{\prime}} \mathrm{J}_{l}\left(\chi \sigma_{s} \sqrt{2 J_{s}}\right) \mathrm{J}_{l^{\prime}}\left(\chi \sigma_{s} \sqrt{2 J_{s}^{\prime}}\right) .
\end{aligned}
$$

In the following we will repeatedly address the case of short bunches when one may put

$$
\varphi \approx-\left(\sigma-\sigma^{\prime}\right) / 2 \beta_{x}^{*}
$$

and obtain an approximation,

$$
L_{l, l^{\prime}}\left(J_{s}, J_{s}^{\prime}\right) \approx \mathrm{J}_{l}\left[\left(\frac{1}{\beta_{x}^{*}}-\chi\right) \sigma_{s} \sqrt{2 J_{s}}\right] \mathrm{J}_{l^{\prime}}\left[\left(\frac{1}{\beta_{x}^{*}}-\chi\right) \sigma_{s} \sqrt{2 J_{s}^{\prime}}\right]
$$

\subsubsection{High synchrotron tune}

Let us first consider the case of large synchrotron tunes, $\left|Q_{s}\right| \gg 1$, when coupling terms in eq.(8.2) can be neglected. Then the Fourier components (8.1) of the distribution function can be expanded as

$$
u_{l}^{( \pm)}(\underline{J}, \theta)=\sum_{i} a_{l, i}^{( \pm)}\left(\underline{J}_{\perp}, \theta\right) v_{i}^{(l)}\left(J_{s}\right)
$$

with $v_{i}^{(l)}$ being the eigenfunctions of the Fredholm operator

$$
\lambda_{\|}^{(i)} v_{i}^{(l)}(J)=\int_{0}^{\infty} L_{l, l}\left(J, J^{\prime}\right) e^{-\left(J+J^{\prime}\right) / 2} v_{i}^{(l)}\left(J^{\prime}\right) d J^{\prime} .
$$

Assuming for a particular term in expansion (8.7) $a_{l}^{( \pm)} \sim \exp (-i \xi \lambda \theta)$ (the index $i$ being omitted for brevity) we arrive at the following eigenvalue problem

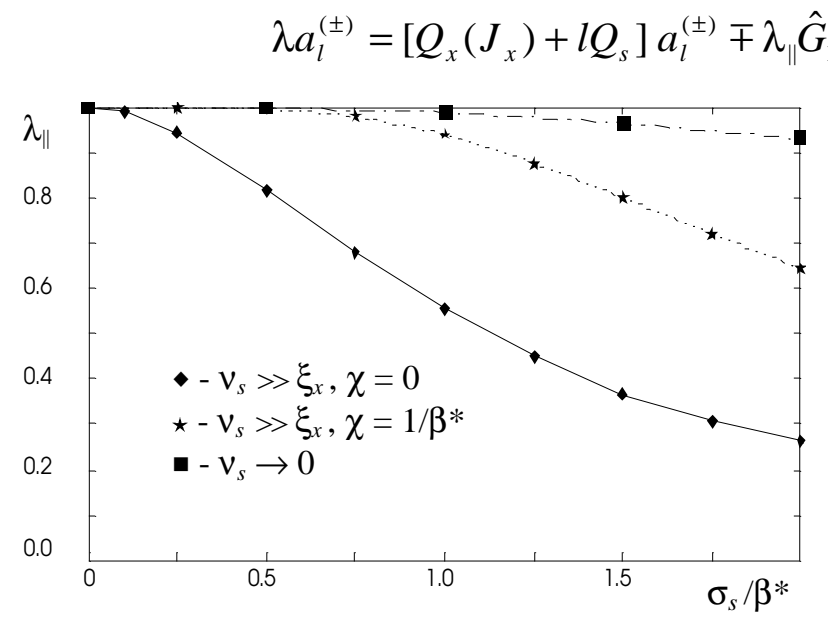

Figure 11. Longitudinal eigenvalue vs. bunch length

$$
\kappa=\left(\frac{1}{\beta_{x}^{*}}-\chi\right) \sigma_{s},
$$

Thus the mentioned weakening of coherence of oscillations due to finite bunch length is determined by the largest longitudinal eigenvalue $\lambda_{\|}$. Comparison with the case of the phase advance redistribution (see eq.(5.23) and Fig.4) shows that for suppression of the discrete modes $\lambda_{\|}<0.25$ is needed.

Making use of approximation (8.6) we obtain for the longitudinal eigenvalue in the case of small bunch length

$$
\lambda_{\|}=e^{-\kappa^{2}} \mathrm{I}_{l}\left(\kappa^{2}\right)
$$

where

$\mathrm{I}_{l}(x)$ is the modified Bessel function of order $l$. The corresponding eigenfunction is

$$
v_{l}(J)=\frac{1}{\sqrt{\lambda_{\|}}} e^{-J / 2} \mathrm{~J}_{l}(\kappa \sqrt{2 J}) .
$$



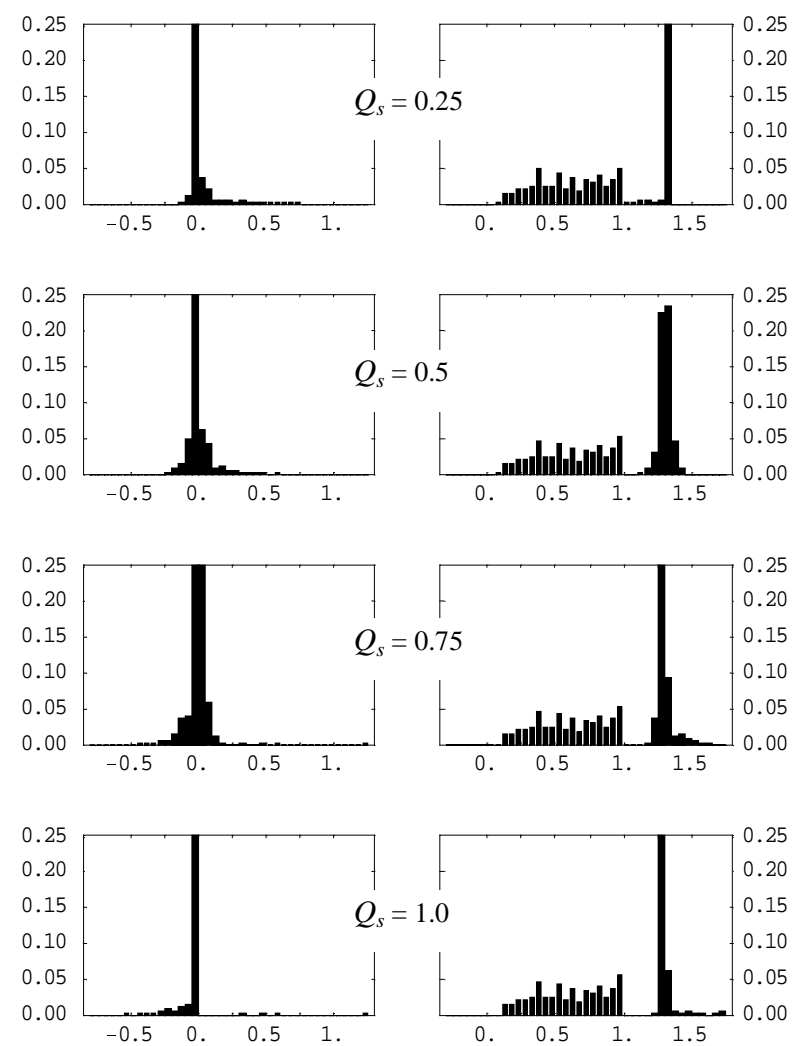

Figure 12. Spectra of $\Sigma$-modes (left) and $\pi$-modes (right) at $\sigma_{s} / \beta^{*}=0.15, \chi=0$ and indicated values of the normalized synchrotron tune.
We see that the discrete modes suppression condition $\left(\lambda_{\|}<0.25\right)$ is naturally satisfied for $l \neq 0$ hence the synchrotron satellites have only continuous spectra, $\boldsymbol{C}_{l}=\left(l Q_{s}, 1+l Q_{s}\right)$. It should be noted that besides (8.10) there is another eigenvalue, $\lambda_{\|}=0$, of infinite (but countable) multiplicity for any $l$, so that the continuous spectra are infinitely degenerate.

In the case of arbitrary bunch length the eigenvalue problem (8.8) has to be solved numerically with the exact kernel (8.6). Fig.11 shows the largest eigenvalue $\lambda_{\|}$for $l=0$ as function of $\sigma_{s} / \beta_{x}{ }^{*}$ at two values of normalized chromaticity: $\chi=0$ and $\chi=1 / \beta_{x}^{*}$ (the corresponding value for LHC is $v_{x}{ }^{\prime}=3$ ). We see that positive chromaticity counteracts the finite bunch length effect on the discrete modes.

\subsubsection{Low synchrotron tune}

In the opposite limit of low synchrotron tunes, $\left|Q_{s}\right| \ll<1$, when coupling to the synchrotron satellites can not be ignored, it is convenient to return back to functions of the synchrotron phase angle

$\psi_{s}$. Then we obtain instead of eq.(2)

$$
\frac{i}{\xi} \frac{\partial}{\partial \theta} u^{( \pm)}=\left(Q_{x}-i Q_{s} \frac{\partial}{\partial \psi_{s}}\right) u^{( \pm)} \mp \hat{G}_{1} \hat{L} u^{( \pm)}
$$

where the longitudinal integral operator was introduced

$$
\hat{L} v\left(J_{s}, \psi_{s}\right)=\int_{0}^{\infty} \int_{0}^{2 \pi} e^{-2 i \varphi-i \chi\left(\sigma-\sigma^{\prime}\right)-\left(J_{s}+J_{s}^{\prime}\right) / 2} v\left(J_{s}^{\prime}, \psi_{s}^{\prime}\right) d J_{s}^{\prime} d \psi_{s}^{\prime} .
$$

In the limit $Q_{s} \rightarrow 0$ the expansion of the type (8.7) is again possible (without index $l$ ), in the eigenfunctions of the operator $\hat{L}$. Its eigenvalue $\lambda_{\|}$does not depend on chromaticity at all (since the corresponding term in the exponent of the kernel can be transformed away) and depends on $\sigma_{s} / \beta_{x}{ }^{*}$ only very weakly.

In the case (8.5) of short bunches we obtain the eigenfunction

$$
v\left(J_{s}, \psi_{s}\right) \approx e^{i\left(1 / \beta^{*}-\chi\right) \sigma-J_{s} / 2}
$$

which corresponds to the eigenvalue $\lambda_{\|}=1$. Again, there is also an infinitely degenerate eigenvalue, $\lambda_{\|}=0$, whose implication will be discussed in more detail in the next subsection.

Numerical results obtained with the exact operator (8.14) are presented in Fig.11 with filled squares. Practically no effect of finite bunch length $\sigma_{s} \leq \beta_{x}^{*}$ on the coherent tuneshift can be seen in the considered limit $Q_{s} \rightarrow 0$. Instead, the beam-beam interaction imposes the 
coherent beam-beam head-tail phase given by the imaginary part of the exponent in eq.(8.15). Its effect on the so-called slow head-tail instability will be discussed in Appendix B.

\subsubsection{Intermediate synchrotron tune}

In the practically most important case of a synchrotron tune comparable with the beam-beam parameter, $Q_{s} \sim 1$, coupling with synchrotron satellites can not be ignored any more and one has to solve the complete Vlasov equation in the form (8.2) or (8.13).

Fig. 12 presents spectra of the dipole $\Sigma$ - and $\pi$-oscillations obtained at $\sigma_{s} / \beta_{x}{ }^{*}=0.15$ with account of coupling with $l= \pm 1, \pm 2$ satellites. The small pikes seen in the $\pi$-mode spectra are a consequence of the limited number of mesh points in the action variables space.

As expected, overlapping by synchrotron sidebands has damping effect on the discrete modes which manifests itself in the line widening. The width of the $\pi$-mode discrete line recovered from the numerical data at $Q_{s}=0.5$ is about 0.03 (in units of $\xi$ ). Calculations with different number of satellites show that it is the first sideband which produces damping in short bunches whereas at $\sigma_{s} / \beta_{x}{ }^{*} \sim 1$ the effect of the second sideband becomes important.

It is possible to proceed further analytically in the case (8.5) of small bunch length. Performing the substitution

$$
u^{( \pm)}\left(\underline{J}, \psi_{s}\right)=e^{i\left(1 / \beta^{*}-\chi\right) \sigma_{\tilde{u}^{( \pm)}}}\left(\underline{J}, \psi_{s}\right)
$$

in eq.(13) with subsequent expansion in the Fourier series in $\psi_{s}$ we get

$$
\frac{i}{\xi} \frac{\partial}{\partial \theta} \tilde{u}_{l}^{( \pm)}=\left[Q_{x}\left(J_{x}\right)+l Q_{s}\right] \tilde{u}_{l}^{( \pm)} \mp \delta_{l, 0} \int_{0}^{\infty} d^{3} J^{\prime} e^{-\sum_{i}\left(J_{i}+J_{i}^{\prime}\right) / 2} G_{1} \tilde{u}_{l}^{( \pm)}+\kappa Q_{s} \sqrt{J_{s}} \frac{\tilde{u}_{l-1}^{( \pm)}+\tilde{u}_{l+1}^{( \pm)}}{\sqrt{2}},
$$

where the (small) coupling parameter $\kappa$ was defined by eq.(8.11). We can see that the (quasi) dipole modes, $l=0$, are directly coupled only to the first satellites. Just one of them with $l=\mp \operatorname{sgn} Q_{s}$ can be retained.

To employ the formalism developed in Appendix A we should give explicit expressions for the eigenfunctions of the diagonal operators.

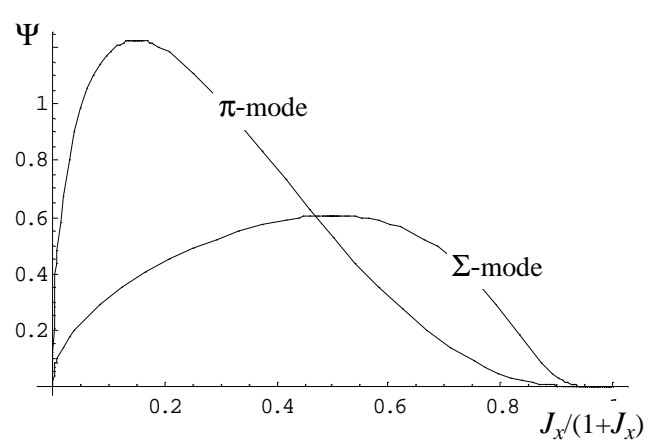

Figure 13. $J_{x}$-dependence of the eigenfunctions corresponding to the discrete modes.

The discrete $\Sigma$-mode (the upper sign in eq.(8.17)) corresponds to the eigenfunction (5.13) with eigenvalue $\lambda_{0}=0$. The eigenfunctions representing discrete $\pi$-modes (of which only that with $\lambda_{0}=1.33$ bears physical significance) have the same exponential dependence on $J_{s}$ (and $J_{y}$ in the flat beam case). The numerically found $J_{x}$ dependence of the eigenfunction corresponding to $\lambda_{0}=1.33$ is shown in Fig.13.

From eq.(8.17) it is clearly seen that modes with $l \neq 0$ have only continuous spectra, $\boldsymbol{C}_{l}=\left(l Q_{s}, 1+l Q_{s}\right)$, each eigenvalue being twice infinitely degenerate since the Hilbert space formed by functions of $\left(J_{y}, J_{s}\right)$ is invariant under the operator $\hat{A}_{l}=Q_{x}\left(J_{x}\right)+l Q_{s}$. The $J_{y}$ - degeneracy is insignificant since the coupling operator

$$
\hat{B}=\kappa Q_{s} \sqrt{J_{s} / 2}
$$

does not remove it. Ignoring it, we may write for the eigenfunctions corresponding to an eigenvalue $\lambda \in \boldsymbol{C}_{l}$ 


$$
\Phi_{\lambda, n}=\frac{1}{\left|d Q_{x} / d J_{x}\right|^{1 / 2}} \delta\left(J_{x}-J_{0}\right) e^{-\left(J_{y}+J_{s}\right) / 2} \mathrm{~L}_{n}\left(J_{s}\right),
$$

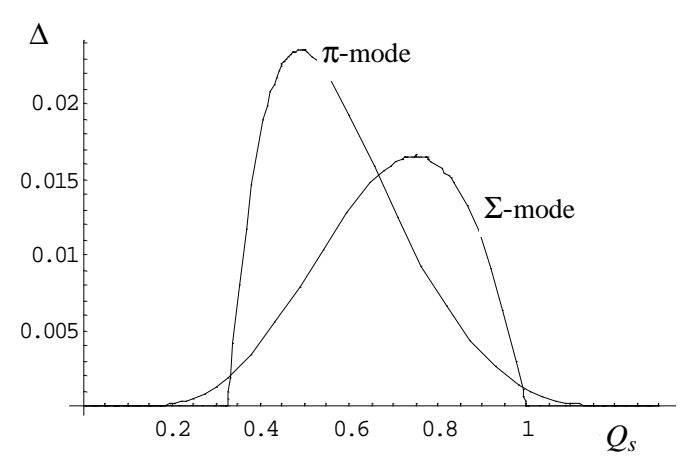

Figure 14. Width of discrete spectral lines in the result of coupling to the first synchrotron sideband at $\kappa=0.15$. where $J_{0}$ is solution of the equation

$$
Q_{x}\left(J_{0}\right)+l Q_{s}=\lambda,
$$

$\mathrm{L}_{n}(x)$ is the Laguerre polynomial of order $n$.

The expression (A.15) for the line width can be easily generalized in the case of degeneracy:

$$
\Delta(\lambda)=\pi \sum_{n=0}^{\infty}\left|\int \Psi_{0} \hat{B} \Phi_{\lambda, n} d^{3} J\right|^{2} .
$$

Inserting the particular expressions (8.18), (8.19) we finally obtain

$$
\Delta(\lambda)=\left.\frac{\pi^{2} D}{8} \kappa^{2} Q_{s}^{2} \frac{\Psi_{0}^{2}}{\left|d Q_{x} / d J_{x}\right|}\right|_{J_{x}=J_{0},}
$$

where the factor $D$ results from the continuum degeneracy,

$$
D=\sum_{n=0}^{\infty}\left[\frac{(-1 / 2)_{n}}{n !}\right]^{2} \approx 1.273,
$$
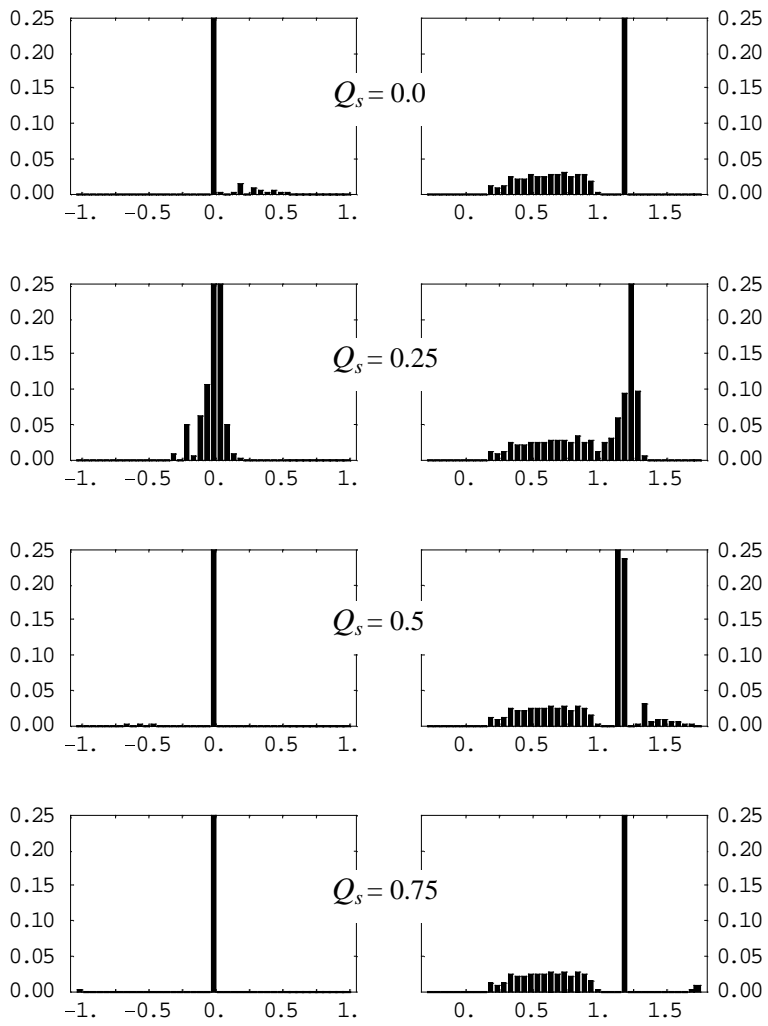

Figure 15. Spectra of $\Sigma$-modes (left) and $\pi$ modes (right) at the reduced crossing angle $2 \omega=1.5$ and indicated values of the normalized synchrotron tune. $(\alpha)_{n}=\alpha(\alpha+1) \ldots(\alpha+n-1),(\alpha)_{0}=1$, being the Pochhammer symbol.

Fig.14 shows the dependence on $Q_{s}$ of the width of the discrete $\pi$ - and $\Sigma$ mode lines obtained from eq.(22) at $\kappa=0.15$. There is a reasonable agreement with the direct solution of the eigenvalue problem for eq.(8.2).

It is noteworthy that calculations in the case of round beams give quite close results to those presented in Fig.14.

\subsection{Effect of finite crossing angle}

A finite crossing angle also reduces the coherent tuneshift and provides coupling with the synchrotron satellites (only evenorder ones in the absence of horizontal offset).

As shown in Ref.[7] the Yokoya factor of the uncoupled dipole $\pi$-mode reaches its minimum value of $Y=1.21$ (in the flat beam case) at the reduced crossing (half) angle $\omega=0.7$ which is quite close to the LHC design value $\omega=0.75$. This fact already facilitates suppression of the coherent $\pi$-mode regardless of the synchrotron tune value. The $\Sigma$-mode is not 
affected by the finite crossing angle alone. However, both discrete lines are noticeably damped being overlapped by the second synchrotron sidebands of the continuum modes.

Fig. 15 shows spectra of the $\Sigma$ - and $\pi$-oscillations at $\omega=0.75$ and several values of $Q_{s}$. We can see that the crossing angle $\omega=0.75$ provides approximately as strong damping as the bunch length does at $\kappa=0.15$.

Since the two damping mechanisms involve different satellites they do not interfere, at least at small values of $\kappa$. The question remains of whether such interference is possible at large bunch lengths when $\kappa \sim 1$.

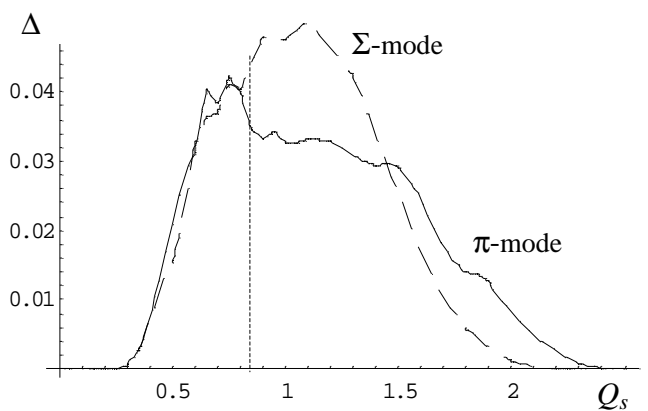

Figure 16. Width of discrete spectral lines at the LHC design parameters and zero chromaticity vs. synchrotron tune with $|\xi|_{\text {IP5 }}=\mathbf{0 . 0 0 2 5}$ used for normalization.

\subsection{Implications for $\mathrm{LHC}$}

In the scheme accepted for LHC there are two diametrically opposite high-luminosity interaction points, IP1 and IP5, at which bunches collide at a crossing angle $2 \alpha=0.3 \mathrm{mrad}$ (the reduced value $2 \omega=1.5)$, in the vertical plane at one IP and in the horizontal plane at the other; the incoherent horizontal tune shifts being correspondingly -0.0031 and $-0.0025[12]$.

The nominal bunches experience 30 longrange parasitic encounters around each IP. Owing to alternating separation, the contribution from these encounters to the total incoherent tune shift is only -0.0004 [12]. As shown in Section 6, they

do not significantly affect the coherent modes as well, which permits us to ignore them altogether.

To further simplify the problem we reduce the dimensionality by assuming flat beams; the effect of the vertical crossing angle on IP1 contribution being taken into account simply by inserting an appropriate correction factor.

With these simplifications we compute the eigenfunctions for uncoupled modes and then employ formula (A.15) to calculate the partial contributions from $l=1,2$ sidebands to the $\pi$-mode line width and from $l=-1,-2$ sidebands to the $\Sigma$-mode line width. Fig.16 shows discrete line widths thus obtained in units of $\mid \xi_{\text {IP } 5}=0.0025$ vs. $Q_{s}$ at $\omega=0.75$ and $\kappa=0.15$. The value of the latter parameter corresponds to zero chromaticity, $\chi=0$.

The design synchrotron tune value, $\left(\left|v_{s}\right|=0.0021\right.$, or $\left.Q_{s}=0.84\right)$ appears to be close to the maximum Landau damping for both modes, the line width being $\approx 0.035|\xi|$ for the $\pi$-mode and $\approx 0.045|\xi|$ for the $\Sigma$-mode. The damping rate $\left(\approx 6 \mathrm{~s}^{-1}\right.$ and $\approx 8 \mathrm{~s}^{-1}$ respectively) is sufficient to suppress the resistive wall instability, whose growth rate at top energy (with account of the magneto-resistive effect) is expected not to exceed $5 \mathrm{~s}^{-1}$ [17].

It is possible to enhance Landau damping by tuning the chromaticity negative. At the given synchrotron tune the contributions to the $\pi$-mode line width due to crossing angle and bunch length are approximately equal, therefore setting $v_{x, y}^{\prime}=-\alpha_{M} R / \beta^{*} \approx-3$ will increase the $\pi$-mode damping rate by at least a factor of 2 (and even more for the $\Sigma$-mode).

Still, to make a margin for other possible instabilities (e.g. due to electron cloud [18]), additional methods for suppression of coherent modes can be implemented, such as tune splitting or phase advance redistribution. 


\section{Summary}

We have considered a number of mechanisms which can suppress discrete spectral lines of coherent beam-beam oscillations.

An efficient method applicable with any number of interaction points is dephasing of betatron oscillations by splitting the tunes in the two beams by an amount larger than the beam-beam tuneshift. However, at a small tune split the discrete spectral lines can reappear due to unbalance in bunch population, which by itself would also have a stabilizing effect. Another drawback of this method is the expansion of the total area occupied by both beams in the tune diagram making it more difficult to avoid incoherent resonances.

In a two-ring machine the tunesplit can be increased so that the beams occupy different cells in the tune diagram. Then another complication may arise due to coherent beam-beam resonances of relatively low order. It can be avoided by further increasing the tunesplit up to an integer value.

With two diametrically opposite IPs it is possible to achieve complete cancellation of the coherent beam-beam effect by means of either odd-integer tunesplit or $\pi / 2$-phase advance redistribution.

In spite of expectations based on the rigid-bunch model, the long-range interactions do not suppress the discrete $\pi$-mode in the plane of separation even when the effect of a large number of interactions is combined in phase. At the same time the $\pi$-mode tuneshift in the other plane may increase quite significantly making this mode more difficult to damp.

In the case of two IPs the effect of the long-range interactions on the coherent modes can be significantly reduced with the help of the alternating crossing proposed earlier to cancel the long-range contribution to incoherent tuneshifts. However, the benefit of alternating crossing may be lost if at the same time one of the two above-mentioned methods for cancellation of the effect of the nominal IPs will be implemented.

Analysis of different mechanisms of synchro-betatron coupling (betatron phase advance variation along the bunch, chromatic tune modulation, crossing angle) shows no drastic reduction in the coherent beam-beam tuneshift.

However, if the synchrotron tune is comparable with the beam-beam parameter, the synchrotron sidebands of the continuum modes can overlap discrete spectral lines providing Landau damping.

In the case of short bunches, $\sigma_{s} / \beta_{x}^{*}<<1$, the Landau damping is produced by the first sideband whereas at $\sigma_{s} / \beta_{x}{ }^{*} \sim 1$ the contribution from the second sideband is also important. Negative chromaticity is found to enhance the finite bunch length effect. The crossing angle contributes to damping by the second sideband.

If the synchrotron tune lies outside the range necessary for these sidebands to overlap the discrete lines, another mechanism of damping the coherent beam-beam modes is possible, the so-called head-tail damping. It is found that when the synchrotron tune is small with respect to the beam-beam parameter, the lattice chromaticity plays no role in the head-tail effect (hence may be negative); the damping rate being determined by the ratio $\sigma_{s} / \beta_{x}{ }^{*}$.

In the case of LHC the design synchrotron tune value renders the Landau damping rate close to the maximum $\left(\approx 6 \mathrm{~s}^{-1}\right.$ for the $\pi$-mode and $\approx 8 \mathrm{~s}^{-1}$ for the $\Sigma$-mode) which is marginally sufficient to suppress the resistive wall instability at top energy. A negative chromaticity $v^{\prime} \sim-3$ provides more than a twofold gain in the damping rate. 


\section{Acknowledgements}

In conclusion the author would like to express his gratitude to the members of the SL AP group for many stimulating discussions of the beam-beam effect which he enjoyed during his visits to CERN, in particular to W.Herr and F.Ruggiero who also carefully read the manuscript and made numerous suggestions for improving it. Also, it is his special pleasure to thank B.Zotter for permanent encouragement and invariable hospitality.

\section{References}

[1] K.Yokoya et al., "Tune Shift of Coherent Beam-Beam Oscillations", KEK Preprint 89-14 (1989); Particle Accelerators, v.27, p.181 (1990).

[2] Y.Alexahin, "On the Landau Damping and Decoherence of Transverse Dipole Oscillations in Colliding Beams", CERN SL-96-064 AP (1996); Particle Accelerators, v.59, p.43 (1998).

[3] A.Hofmann, "Beam-Beam Modes for Two Beams with Unequal Tunes", in: Proc. LHC 99 Workshop, CERN-SL-99-039 AP, Geneva, 1999, pp.56-58.

[4] Y.Alexahin, "Eigenmodes of Coherent Oscillations in Colliding Beams", ibid, pp.41-44.

[5] A.Temnykh, J.Welch, "Crossing Angles at CESR, Experiments and Experience", in: Proc. VII Advanced ICFA Beam Dynamics Workshop, Dubna, 1995, pp.48-54.

[6] K.Hirata, H.Moshammer and F.Ruggiero, "Synchro-Beam Interaction", CERN SL-AP/90-92 (1992), Particle Accelerators, v.40, p.205 (1993).

[7] Y.Alexahin, "Coherent Synchro-Betatron Oscillations in Colliding Beams", Frascati Physics Series, v.X, p.255 (1998).

[8] L.Michelotti, "Intermediate Classical Dynamics with Applications to Beam Physics". J.Wiley, 1995.

[9] A.W.Chao, "Physics of Collective Beam Instabilities in High Ehergy Accelerators", J.Wiley, 1993.

[10] W.Herr, private communication.

[11] J.Gareyte, "Beam-Beam Design Criteria for LHC", in: Proc. LHC'99 Workshop, CERNSL-99-039 AP, Geneva, 1999, pp.28-32.

[12] H.Grote, "Beam-Beam Effects in the LHC", ibid, pp.59-62.

[13] W.Herr, J.Miles, "Optimizing the LHC Interaction Region to Obtain the Highest Possible Luminosity", in: Proc. EPAC'96, Sitges, pp.424-426 (1996).

[14] P.Zenkevich, K.Yokoya, "Landau Damping of Coherent Beam-Beam Oscillations", KEK Preprint 92-116 (1992); Particle Accelerators, v.40, p.229 (1992).

[15] /afs/cern.ch/user/a/alexahin/public/mathem/cobbres.nb

[16] Y.Alexahin, M.P.Zorzano, "Excitation of Coherent Beam-Beam Resonances for Beams with Unequal Tunes in the LHC", LHC Project Note 226 (2000).

[17] F.Ruggiero, "Single-Beam Collective Effects in the LHC", CERN SL/95-09 (AP) (1995).

[18] F.Zimmermann, "Electron-cloud Simulations for the LHC Straight Sections", LHC Project Note 201 (1999).

[19] P.Zenkevich, "Process of Relaxation of Transverse Dipole Oscillations in a Circular Beam of Charged Particles", Preprint ITEP No.951, Moscow, 1972 (in Russian).

[20] L.Landau, "On the Vibration of the Electronic Plasma", J.Phys. (USSR), v.10, No.1, pp.25-34 (1946).

[21] N.G.Van Kampen, "On the Theory of Stationary Waves in Plasmas", Physica, v.21, No.12, pp.949-963 (1955).

[22] V.Bharadwaj et al., "Fermilab Collider Run 1b Accelerator Performance", FERMILABTM-1970 (1996). 


\section{Appendix A. Landau damping by sidebands}

Here we obtain the formula for the width which a discrete spectral line (of dipole oscillations) obtains due to being overlapped by bounded continuous spectrum (of a sideband).

The spectrum boundedness ${ }^{4}$ presents an important distinction from the classical case of plasma oscillations. We will treat the problem following both the original method of Landau [20] and a more general approach proposed by Van Kampen [21].

Let us consider the equation

$$
\frac{\partial}{\partial \theta} \mathbf{f}=-i \hat{A} \cdot \mathbf{f}
$$

for vector function of variable $J$

$$
\mathbf{f}=\left(\begin{array}{l}
f_{1} \\
f_{2}
\end{array}\right)
$$

where

$$
\hat{A}=\left(\begin{array}{ll}
\hat{A}_{0} & \hat{B} \\
\hat{B}^{*} & \hat{A}_{1}
\end{array}\right)
$$

is a matrix of operators acting in the Gilbert space of functions of $J, \hat{B}$ presents a (small) coupling perturbation, asterisk means Hermitian conjugation. We assume the diagonal operators to be self-adjoint, $\hat{A}_{i}^{*}=\hat{A}_{i} ; \hat{A}_{0}$ to have a discrete eigenvalue $\lambda_{0}$ well separated from the rest of its spectrum $\boldsymbol{R}$ (the criterion will be clear afterwards):

$$
\hat{A}_{0} \Psi_{0}=\lambda_{0} \Psi_{0},
$$

and $\hat{A}_{1}$ to have a continuous spectrum $C=\left(\lambda_{\min }, \lambda_{\max }\right)$ overlapping $\lambda_{0}\left(\lambda_{\min }<\lambda_{0}<\lambda_{\max }\right)$ :

$$
\hat{A}_{1} \Phi_{\lambda}=\lambda \Phi_{\lambda}, \quad \lambda \in C
$$

Eigenfunctions of operators $\hat{A}_{0,1}$ satisfy the following orthonormality conditions

$$
\left(\Psi_{0}, \Psi_{0}\right) \equiv \int\left|\Psi_{0}\right|^{2} d J=1, \quad\left(\Phi_{\lambda}, \Phi_{\lambda^{\prime}}\right)=\delta\left(\lambda-\lambda^{\prime}\right)
$$

With the help of these eigenfunctions we can look for the solution of eq.(A.1) in the form

$$
\mathbf{f}=\left(\begin{array}{c}
a_{0} \Psi_{0}+\int_{R} a_{\mu} \Psi_{\mu} d w(\mu) \\
\int_{C} b_{\mu} \Phi_{\mu} d \mu
\end{array}\right),
$$

Being interested in eigenvalues of $\hat{A}$ in the vicinity of $\lambda_{0}$ we may neglect the contribution of the Stieltjes integral over $\boldsymbol{R}$ owing to the assumption made above.

\footnotetext{
${ }^{4}$ As far as the author is aware of, the case of bounded spectrum was first treated by P.Zenkevich [19].
} 


\section{A.1 Landau recipe}

Let us follow the classical work of Landau [20] and consider the initial value problem for eq.(1), choosing the particular initial conditions

$$
\left.f_{0}\right|_{\theta=0}=\Psi_{0},\left.\quad f_{1}\right|_{\theta=0}=0,
$$

or, for the expansion coefficients

$$
\begin{array}{ll}
a_{0}(0)=1, & a_{\lambda}(0)=0, \quad \lambda \in \boldsymbol{R}, \\
b_{\lambda}(0)=0, & \lambda \in \boldsymbol{C},
\end{array}
$$

Performing the Laplace transformation

$$
a_{0 p}=\int_{0}^{\infty} e^{-p \theta} a_{0}(\theta) d \theta .
$$

we obtain from eq.(A.1) the system

$$
\begin{aligned}
& \left(p+i \lambda_{0}\right) a_{0 p}+i \int_{C}\left(\Psi_{0}, \hat{B} \Phi_{\mu}\right) b_{\mu p} d \mu=1, \\
& (p+i \lambda) a_{\lambda p}+i \int_{C}\left(\Psi_{\lambda}, \hat{B} \Phi_{\mu}\right) b_{\mu p} d \mu=0, \quad \lambda \in \boldsymbol{R}, \\
& (p+i \lambda) b_{\lambda p}+i\left(\Phi_{\lambda}, \hat{B}^{*} \Psi_{0}\right) a_{0 p}+i \int_{\boldsymbol{R}}\left(\Phi_{\lambda}, \hat{B}^{*} \Psi_{\mu}\right) a_{\mu p} d \mu=0, \quad \lambda \in \boldsymbol{C}
\end{aligned}
$$

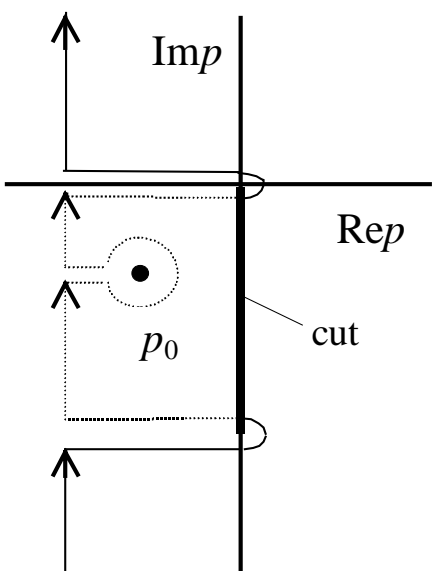

Figure 17. Contour of integration.

Neglecting the contribution from $\boldsymbol{R}$ to the last of eqs.(A.10) we can write for the solution of eq.(A.1)

$$
a_{0}(\theta)=\frac{1}{2 \pi i} \int_{\varepsilon-i \infty}^{\varepsilon+i \infty} \frac{e^{p \theta}}{D(p)} d p,
$$

where $\varepsilon$ is a constant larger than the real part of any singularity of the inverse of the function

$$
\begin{aligned}
& D(p)=i p-\lambda_{0}+\int_{C} \frac{\left|B_{\mu}\right|^{2}}{\mu-i p} d \mu, \\
& B_{\mu} \equiv\left(\Psi_{0}, \hat{B} \Phi_{\mu}\right) .
\end{aligned}
$$

The integral over continuous spectrum (hence $D(p)$ on the whole) is analytical everywhere in the complex domain of $p$ except for the cut $\left(-i \lambda_{\max },-i \lambda_{\min }\right)$ which ends are logarithmic branching points. It is easy to verify that $D(p)$ has no zeros on the main list. At the same time, its analytic continuation across the cut from the right half-plane to $\operatorname{Re} p<0$ has a zero.

We can obtain the exact expression by reducing (A.11) to an integral around the cut [19]. However, it will be more instructive to use the analytic continuation technique which has a significant heuristic potential and easily provides solution in even more complicated cases (e.g. of degeneracy of the continuous spectrum).

According to the Sohotsky-Plemmel formula we have for $p$ immediately to the right of the cut

$$
\int_{C}^{\left|B_{\mu}\right|^{2}} \frac{{ }^{2}}{\mu-i p} d \mu=\text { p.v. } \int_{C} \frac{\left|B_{\mu}\right|^{2}}{\mu+\operatorname{Im} p} d \mu+\pi i\left|B_{-\operatorname{Im} p}\right|^{2},
$$


where p.v. means the principal value of the integral, and may use this equality as an approximation for the analytic continuation of $D(p)$ to $\operatorname{Re} p<0$.

Now assuming that solution of the equation

$$
\tilde{\lambda}_{0}=\lambda_{0}-\text { p.v. } \int_{C} \frac{\left|B_{\mu}\right|^{2}}{\mu-\tilde{\lambda}_{0}} d \mu
$$

still belongs to $C$ we obtain a zero of $D(p)$ at

$$
p=p_{0} \equiv-i \tilde{\lambda}_{0}-\Delta\left(\tilde{\lambda}_{0}\right), \quad \Delta(\lambda) \equiv \pi\left|B_{\lambda}\right|^{2} .
$$

To evaluate the integral in (A.11) let us deform the path of integration as shown in Fig.17, threading it in and out of the cut and encircling the pole at $p=p_{0}$. Taking the part of the integral given by the residue at the pole,

$$
a_{0}(\theta) \approx e^{p_{0} \theta},
$$

we get finally the dipole component of the solution of eq.(A.1) in the form

$$
f_{0}(\theta) \approx a_{0}(\theta) \Psi_{0} \approx e^{-i \tilde{\lambda}_{0} \theta-\Delta\left(\tilde{\lambda}_{0}\right) \theta} \Psi_{0}
$$

and can see Landau damping of the discrete mode with decrement $\Delta\left(\tilde{\lambda}_{0}\right)$.

There are certain drawbacks in the above derivation. First, it is not easy to give an estimation of the neglected contribution from the parts of the contour going to and from the ends of the cut on different lists (see Fig.17).

Second, there is an apparent contradiction: eq.(A.16) suggests $\hat{A}$ have a complex eigenvalue, $i p_{0}=\tilde{\lambda}_{0}-i \Delta\left(\tilde{\lambda}_{0}\right)$, whereas this operator is self-adjoint by assumption and may have only real eigenvalues.

\section{A.2 Van Kampen approach}

Let us look for eigenfunctions $\mathbf{F}_{\lambda}$ of operator $\hat{A}$ corresponding to eigenvalues $\lambda$ in the vicinity of $\lambda_{0}$ in the form (A.7) again neglecting the contribution from $\boldsymbol{R}$. Then the expansion coefficients satisfy the system of homogeneous equations

$$
\begin{aligned}
& \left(\lambda-\lambda_{0}\right) a_{0}-\int_{C} B_{\mu} b_{\mu} d \mu=0, \\
& (\lambda-\mu) b_{\mu}-\bar{B}_{\mu} a_{0}=0, \quad \mu \in C
\end{aligned}
$$

which may have nontrivial solution if

$$
\lambda-\lambda_{0}+\int_{C} \frac{\left|B_{\mu}\right|^{2}}{\mu-\lambda} d \mu=0 .
$$

This equation looks very much like equation $D(p)=0$ after substitution $p \rightarrow-i \lambda$ in (A.12) but has an absolutely different meaning. Eq.(A.12) defines $D(p)$ with the integration rule given by the Sohotsky-Plemmel formula (A.13) whereas eq.(A.19) defines the rule of evaluation of the improper integral for a particular value of $\lambda \in \boldsymbol{C}$.

Generally we may write

$$
\frac{1}{\mu-\lambda}=p \cdot v \cdot \frac{1}{\mu-\lambda}+h(\lambda) \delta(\mu-\lambda),
$$

where $h(\lambda)$ is an arbitrary function. To satisfy eq.(A.19) we should choose it as 


$$
h(\lambda)=-\frac{1}{\left|B_{\lambda}\right|^{2}}\left(\lambda-\lambda_{0}+\text { p.v. } \int_{C} \frac{\left|B_{\mu}\right|^{2}}{\mu-\lambda} d \mu\right) .
$$

We are interested in the dependence $a_{0}(\lambda)$ which gives the dipole component of the eigenmodes of operator $\hat{A}$ as a function of eigenvalue $\lambda \in \boldsymbol{C}$. To find it we make use of the orthonormality condition for vector eigenfunctions belonging to continuous spectrum

$$
\begin{aligned}
\left(\mathbf{F}_{\lambda}, \mathbf{F}_{\lambda^{\prime}}\right) & =a_{0}(\lambda) a_{0}\left(\lambda^{\prime}\right)\left[1+\int_{C} \frac{k \hat{B}>\left._{\mu}\right|^{2}}{(\mu-\lambda)\left(\mu-\lambda^{\prime}\right)} d \mu\right]= \\
& =\delta\left(\lambda-\lambda^{\prime}\right),
\end{aligned}
$$

where eqs.(A.6), (A.7) and (A.18) were used to obtain the first equality. Making notice that

$$
\begin{aligned}
& \int_{\lambda-\eta}^{\lambda+\eta} \frac{d \lambda^{\prime}}{\mu-\lambda^{\prime}}=\ln \left|\frac{\mu-\lambda+\eta}{\mu-\lambda-\eta}\right|+\sigma\left[\eta^{2}-(\mu-\lambda)^{2}\right] h(\mu), \\
& \lim _{\eta \rightarrow 0} \frac{1}{\mu-\lambda} \ln \left|\frac{\mu-\lambda+\eta}{\mu-\lambda-\eta}\right|=\pi^{2} \delta(\mu-\lambda),
\end{aligned}
$$

where $\sigma(x)=1$ for $x \geq 0$ and $\sigma(x)=0$ for $x<0$, and performing integration of eq.(A.22) by $\lambda^{\prime}$ in the limits $(\lambda-\eta, \lambda+\eta)$ where $\eta$ is an infinitesimal value, we have

$$
\int_{\lambda-\eta}^{\lambda+\eta}\left(\mathbf{F}_{\lambda}, \mathbf{F}_{\lambda^{\prime}}\right) d \lambda^{\prime}=1=a_{0}^{2}(\lambda)\left|B_{\lambda}\right|^{2}\left[\pi^{2}+h^{2}(\lambda)\right],
$$

or, recalling eq.(A.21),

$$
a_{0}^{2}(\lambda)=\frac{\left|B_{\lambda}\right|^{2}}{\pi^{2}\left|B_{\lambda}\right|^{4}+\left(\lambda-\lambda_{0}+\text { p.v. } \int_{C} \frac{\left|B_{\mu}\right|^{2}}{\mu-\lambda} d \mu\right)^{2}} .
$$

We see that the position of the peak and its width are very similar to those obtained by the first method. But there is some difference in the meaning of coefficients $a_{0}(\lambda)$ and $a_{0}(\theta)$ which needs clarification.

\section{A.3 Correspondence of the two methods}

Let us again consider the initial conditions (A.8). Now we solve eq.(A.1) by expansion in the eigenfunctions $\mathbf{F}_{\lambda}$ of operator $\hat{A}$ :

$$
\mathbf{f}(\theta)=\int_{C} c_{\lambda}(\theta) \mathbf{F}_{\lambda} d \lambda, \quad c_{\lambda}(0)=\left(\mathbf{f}(0), \mathbf{F}_{\lambda}\right)=a_{0}(\lambda),
$$

and obtain for the dipole component

$$
f_{0}(\theta)=\int_{C} c_{\lambda}(\theta) a_{0}(\lambda) d \lambda \Psi_{0}=\int_{C} e^{-i \lambda \theta} a_{0}^{2}(\lambda) d \lambda \Psi_{0}
$$

Comparing this result with eq.(A.17) we get the sought relation

$$
a_{0}(\theta)=\int_{C} e^{-i \lambda \theta} a_{0}^{2}(\lambda) d \lambda
$$

We can proceed further assuming the width $\Delta(\lambda)=\pi\left|B_{\lambda}\right|^{2}$ small and slowly varying: $\Delta\left(\lambda_{0}\right) \ll \min \left(\lambda_{\max }-\lambda_{0}, \lambda_{0}-\lambda_{\min }\right), \Delta^{\prime}\left(\lambda_{0}\right) \ll 1$. Then we may extend integration limits in (A.28) to $\pm \infty$ and, regarding $\Delta(\lambda)$ as a constant, obtain (A.17). 


\section{Appendix B. Beam-beam effect on the slow head-tail instability}

The coherent beam-beam head-tail phase given by eq.(8.15) can completely change the picture of the so-called slow head-tail instability (see e.g. Ref.[9]). Here we consider only the effect of finite bunch length and chromaticity in the absence of crossing angle.

In the presence of external elements we should add the corresponding term to the Liouville equation (4.3):

$$
\frac{\partial}{\partial \theta} F^{(k)}+\left[F^{(k)}, K^{(k)}\right]=\underline{\dot{I}}^{(\mathrm{ext})} \cdot \underline{\varepsilon}^{-1} F_{0},
$$

where the invariant (in the absence of perturbations) action $\underline{I}$ to the first order in the beambeam parameter and chromaticity coincides with the original action. Therefore its derivative can be expressed via the wake fields as

$$
\dot{I}_{x}^{(\mathrm{ext})}=\beta_{x} p_{x} \dot{p}_{x}^{(\mathrm{ext})}=-\beta_{x} p_{x} \frac{e^{2} N_{k}}{2 \pi} \int d^{3} I^{\prime} d^{3} \psi^{\prime} W_{1}\left(\sigma-\sigma^{\prime}\right) x^{\prime} F_{1}^{(k)}\left(I^{\prime}, \psi^{\prime}, \theta\right)
$$

(the case of horizontal dipole oscillations, $m_{x}=1, m_{y}=0$, assumed).

Let us perform Fourier expansion in the angle variables of transverse motion but retain dependence on the synchrotron phase angle $\psi_{s}$, the scalar product being now defined as

$$
(\mathbf{f}, \mathbf{g})=\frac{1}{2 \pi} \int\left(\bar{f}^{(1)} g^{(1)}+\bar{f}^{(2)} g^{(2)}\right) d^{3} J d \psi_{s} .
$$

Implementing (with the said exception) the transformations which lead to eq.(5.5) and assuming equal single particle tunes we may write for the external elements contribution to the Vlasov operator

$$
\xi \hat{A}^{(\mathrm{ext})}=\frac{\beta_{x}\left(1+i \alpha_{x}\right) e^{2}}{8 \pi^{2}} \Psi_{0}(\underline{J}) \int d^{3} J^{\prime} d \Psi_{s}^{\prime} \Psi_{0}\left(\underline{J^{\prime}}\right) W_{1}\left(\sigma-\sigma^{\prime}\right) e^{-i \chi\left(\sigma-\sigma^{\prime}\right)}\left(\begin{array}{cc}
N_{1} & 0 \\
0 & N_{2}
\end{array}\right)
$$

where expressions (3.17) for $x$-coordinate and momentum were used, $\Psi_{0}(\underline{J})$ is given by eq.(5.13). The term with the Twiss parameter $\alpha_{x}$ vanishes on average over the machine circumference.

At energies in the $\mathrm{TeV}$ range the (complex) tuneshift induced by external impedances is small compared to the beam-beam tuneshift. Therefore i) operator (B.4) can be considered as a small perturbation, ii) only perturbation of discrete modes which are not already Landau damped presents interest, iii) the beam-beam modes with $m_{s} \neq 0$ are stable since they have no discrete eigenvalues.

As follows from the analysis in Section 8.1 the discrete modes are not Landau damped at either small or large values of the synchrotron tune compared to the beam-beam parameter: $\left|Q_{s}\right| \equiv\left|v_{s} / \xi\right|<1$ or $\left|Q_{s}\right| \geq 1$. In both cases the eigenfunctions can be factorized in the transverse and longitudinal parts, the latter being given by eq.(8.15) in the first case and eq.(8.12) with $l=0$ in the second case.

Applying the first order formula for perturbation of eigenvalues of a linear operator we obtain 


$$
\begin{aligned}
\xi \lambda^{(1)}=\left(\boldsymbol{\Psi}_{\lambda}, \xi \hat{A}^{(\text {(ext) })} \boldsymbol{\Psi}_{\lambda}\right)= & \frac{e^{2} N_{2}}{16 \pi^{3}} r_{b b} \int d J_{s} d \Psi_{s} d J_{s}^{\prime} d \psi_{s}^{\prime} e^{-\left(J_{s}+J_{s}^{\prime}\right)} \beta_{x} W_{1}\left(\sigma-\sigma^{\prime}\right) \times \\
& \times\left\{\begin{array}{cl}
\frac{\mathrm{J}_{0}\left(\kappa \sqrt{J_{s}}\right) \mathrm{J}_{0}\left(\kappa \sqrt{J_{s}^{\prime}}\right)}{e^{-\kappa^{2}} \mathrm{I}_{0}\left(\kappa^{2}\right)} e^{-i \chi\left(\sigma-\sigma^{\prime}\right)}, & \left|Q_{s}\right|>1 \\
e^{-i\left(\sigma-\sigma^{\prime}\right) / \beta^{*}}, & \left|Q_{s}\right|<<1
\end{array}\right.
\end{aligned}
$$

where factor $r_{b b}$ is defined by eq.(5.15).

We will not discuss here the head-tail instability in detail and just want to notice that in the case of low synchrotron tunes, $\left|Q_{s}\right| \ll<1$, the lattice chromaticity is excluded from the stability criterion $(\xi \operatorname{Im} \lambda<0)$, its sign playing no role as observed in collision at the Tevatron [22]. Instead, the head-tail phase imposed by the beam-beam interaction of finite length bunches insures damping of the discrete dipole modes ${ }^{5}$.

At intermediate $Q_{s}$ values when damping by synchrotron sidebands comes into force it is even advantageous to make the chromaticity negative (with the absolute value compatible with the single-particle stability) so that it adds up to the damping effect of the finite bunch length. However, at $\left|Q_{s}\right|>1$, when Landau damping by synchrotron sidebands is switched off, the chromaticity should be positive.

\footnotetext{
${ }^{5}$ Let us remind the general properties of the transverse wake function [9]: $W_{1}(z)=0$ at $\mathrm{z} \geq 0$ and $W_{1}(z)<0$ over some distance at $z<0$.
} 